\title{
Influence of mechanical stress in semiconductor heterostructure on density of $p-n$-junctions
}

\author{
E. L. Pankratov
}

Received: 1 August 2011/Accepted: 12 October 2011/Published online: 11 November 2011

(C) The Author(s) 2011. This article is published with open access at Springerlink.com

\begin{abstract}
It has been recently shown that inhomogeneity of semiconductor heterostructure and optimization of annealing time leads to decrease depth of $p-n$-junctions and to increased homogeneity of dopant distribution in doped area. It has been also recently shown that mechanical stress in two-layer heterostructure (substrate and epitaxial layer) changes dopant distribution in heterostructure in directions, which are perpendicular to interface between layers of heterostructure, in comparison with unstressed sample. In this paper we consider an alternative approach to increase density of $p-n$-junctions in the same heterostructure by using overlayer. The overlayer leads to additional mechanical stress. The stress gives us possibility to increase density of $p-n$-junctions in the heterostructure.
\end{abstract}

Keywords Diffusion-junction rectifier .

Increasing of density of rectifiers - Mechanical stress

\section{Introduction}

One of actual problems of the solid-state electronics is increasing of density of elements of integrated circuits (IC) (Grebene 1983; Gotra 1991; Lachin and Savelov 2001). To increase of density of elements of IC it has been recently elaborated nearsurficial (laser and microwave) types of annealing and inhomogenous distributions of defects in doped areas of elements of IC. Recently (see, for example,

E. L. Pankratov $(\square)$

Nizhny Novgorod State University of Architecture and Civil

Engineering, 65 Il'insky street, Nizhny Novgorod 603950,

Russia

e-mail: elp2004@mail.ru
Pankratov 2005; Pankratov and Spagnolo 2005), an alternative approach to decrease of depth of $p-n$-junctions has been introduced. To use the approach we consider a semiconductor heterostructure $(\mathrm{H})$, which consists of a substrate (S) with known type of conductivity ( $n$ or $p$ ) and epitaxial layer (EL). In the EL a dopant has been infused. The dopant produced the second type of conductivity ( $p$ or $n)$. Further, the dopant has been annealed to produce $p-n$ junction near interface between layers of $H$. In this situation sharpness of the $p-n$-junction increases and at the same time homogeneity of dopant distribution in doped area also increases in comparison with $p-n$-junction in homogenous sample. In the Ref. (Pankratov 2010) it has been shown that mechanical stress (the stress is consequence of mismatching in the lattice strain) could change density of $p-n$-junctions on the surface of $H$. In this paper we consider a $\mathrm{H}$, which consist of $\mathrm{S}$ with known type of conductivity ( $n$ or $p$ ) and EL (see Fig. 1). A dopant has been infused in the EL to produce a $p-n$-junctions near interface between layers of $\mathrm{H}$. Before annealing of dopant the EL has been overgrown by an overlayer (OL) with low temperature. During the overgrowth dopant diffusion is negligible. Further annealing of dopant has been done. During the annealing dopant diffusion achieves the interface between EL and S. In this situation density of $p-n$ junctions in EL increases in comparison with situation in Pankratov (2010). The main aim of the present paper is the determination of conditions to increase density of $p-n$ junctions in EL.

\section{Method of solution}

To solve our aims let us determine spatiotemporal distribution of dopant concentration. We determine the 


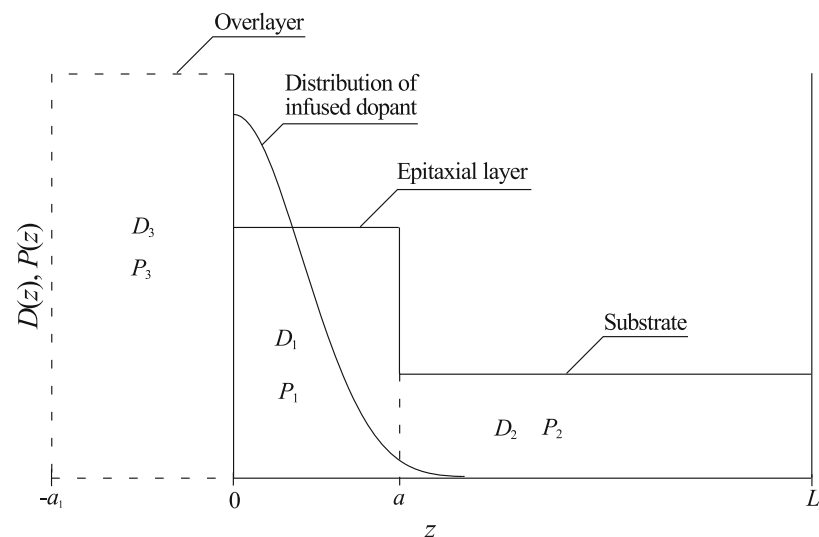

Fig. 1 Heterostructure, which consists of overlayer $\left(z \in\left[-a_{1}, 0\right]\right)$, epitaxial layer $(z \in[0, a])$ and substrate $\left(z \in\left[-a, L_{z}\right]\right)$. The figure also illustrates initial (before starting of annealing) distribution of dopant

distribution by solving the second Fick's law (Grebene 1983; Gotra 1991; Lachin and Savelov 2001)

$$
\begin{aligned}
& \frac{\partial C(x, y, z, t)}{\partial t}=\frac{\partial}{\partial x}\left[D \frac{\partial C(x, y, z, t)}{\partial x}\right] \\
& +\frac{\partial}{\partial y}\left[D \frac{\partial C(x, y, z, t)}{\partial y}\right]+\frac{\partial}{\partial z}\left[D \frac{\partial C(x, y, z, t)}{\partial z}\right] \\
& +\Omega \frac{\partial}{\partial x}\left[\frac{D_{S}}{k T} \nabla_{S} \mu(x, y, z, t) \int_{0}^{L_{z}} C(x, y, W, t) \mathrm{d} W\right] \\
& +\Omega \frac{\partial}{\partial y}\left[\frac{D_{S}}{k T} \nabla_{S} \mu(x, y, z, t) \int_{0}^{L_{z}} C(x, y, W, t) \mathrm{d} W\right]
\end{aligned}
$$

with boundary and initial conditions

$$
\begin{aligned}
& \left.\frac{\partial C(x, y, z, t)}{\partial x}\right|_{x=0}=0,\left.\quad \frac{\partial C(x, y, z, t)}{\partial x}\right|_{x=L_{x}}=0, \\
& \left.\frac{\partial C(x, y, z, t)}{\partial y}\right|_{y=0}=0,\left.\quad \frac{\partial C(x, y, z, t)}{\partial y}\right|_{y=L_{y}}=0, \\
& \left.\frac{\partial C(x, y, z, t)}{\partial z}\right|_{z=0}=0,\left.\quad \frac{\partial C(x, y, z, t)}{\partial z}\right|_{z=L_{z}}=0 \\
& C(x, y, z, 0)=f_{C}(x, y, z) .
\end{aligned}
$$

Here $C(x, y, z, t)$ is spatiotemporal distribution of dopant concentration; $\Omega$ is atomic volume; $\nabla_{S}$ is superficial gradient; $\int_{0}^{L_{z}} C(x, y, W, t) \mathrm{d} W$ is surficial concentration of dopant; $\mu(x, y, z, t)$ is the chemical potential; $D$ and $D_{S}$ are the volumetric and the surficial (due to mechanical stress) dopant diffusion coefficients. Values of dopant diffusion coefficients depend on properties of materials of layers in $\mathrm{H}$, on rate of heating and cooling of $\mathrm{H}$ and on spatiotemporal distribution of dopant concentration. Concentrational dependence of diffusion coefficient could be approximated by the following functions (Gotra 1991):
$D=D_{L}(x, y, z, T)\left[1+\xi \frac{C^{\gamma}(x, y, z, t)}{P^{\gamma}(x, y, z, T)}\right]$,

$D_{S}=D_{S L}(x, y, z, T)\left[1+\xi_{S} \frac{C^{\gamma}(x, y, z, t)}{P^{\gamma}(x, y, z, T)}\right]$.

Here $P(x, y, z, T)$ is limit of solubility of dopant in $\mathrm{H}$; $D_{L}(x, y, z, T)$ and $D_{S L}(x, y, z, T)$ are the diffusion coefficients for low-level of doping; parameter $\gamma$ depends on properties of materials of $\mathrm{H}$ and could be integer usually in the interval $\gamma \in[1,3]$ (Gotra 1991). Chemical potential could be determine by the following relation (Zhang and Bower 1999):

$\mu(x, y, z, t)=E(z) \Omega \sigma_{i j} \frac{u_{i j}(x, y, z, t)+u_{j i}(x, y, z, t)}{2}$,

where $E(z)$ is Young modulus; $\sigma_{i j}$ is the stress tensor; $u_{i j}=$ $\frac{1}{2}\left(\frac{\partial u_{i}}{\partial x_{j}}+\frac{\partial u_{j}}{\partial x_{i}}\right)$ is the tensor of strain; $u_{i}$ and $u_{j}$ are the components $u_{x}(x, y, z, t), u_{y}(x, y, z, t)$ and $u_{z}(x, y, z, t)$ of the displacement vector $\vec{u}(x, y, z, t) ; x_{i}$ and $x_{j}$ are coordinates $x, y$ and $z$. The Eq. 3 could be transform in the form (see Zhang and Bower 1999)

$$
\begin{aligned}
\mu(x, y, z, t)= & E(z) \frac{\Omega}{2}\left[\frac{\partial u_{i}(x, y, z, t)}{\partial x_{j}}+\frac{\partial u_{j}(x, y, z, t)}{\partial x_{i}}\right] \\
& \times\left\{\frac{1}{2}\left[\frac{\partial u_{i}(x, y, z, t)}{\partial x_{j}}+\frac{\partial u_{j}(x, y, z, t)}{\partial x_{i}}\right] .\right. \\
& -\epsilon_{0} \delta_{i j}+\frac{\sigma(z) \delta_{i j}}{1-2 \sigma(z)}\left[\frac{\partial u_{k}(x, y, z, t)}{\partial x_{k}}-3 \epsilon_{0}\right] \\
& \left.-K(z) \chi(z)\left[T(x, y, z, t)-T_{r}\right]\right\} .
\end{aligned}
$$

Here $\sigma$ is the Poisson ratio. $\epsilon_{0}=\left(a_{S}-a_{E L}\right) / a_{E L}$ is the mismatch strain. $a_{S}$ and $a_{E L}$ are free lattice spacing of the substrate and film, respectively. $K$ is the modulus of comprehensive compression. $\chi$ is the coefficient of the thermal expansion. $T_{r}$ is the equilibrium temperature. Let us consider the equilibrium temperature as the room temperature. Components of the displacement vector could be determined from the following system of equation (see, for example, Landau and Lefshits 2001):

$\left\{\begin{array}{l}\rho(z) \frac{\partial^{2} u_{x}(x, y, z, t)}{\partial t^{2}}=\frac{\partial \sigma_{x x}(x, y, z, t)}{\partial x}+\frac{\partial \sigma_{x y}(x, y, z, t)}{\partial y}+\frac{\partial \sigma_{x z}(x, y, z, t)}{\partial z} \\ \rho(z) \frac{\partial^{2} u_{y}(x, y, z, t)}{\partial t^{2}}=\frac{\partial \sigma_{y x}(x, y, z, t)}{\partial x}+\frac{\partial \sigma_{y y}(x, y, z, t)}{\partial y}+\frac{\partial \sigma_{y z}(x, y, z, t)}{\partial z} \\ \rho(z) \frac{\partial^{2} u_{z}(x, y, z, t)}{\partial t^{2}}=\frac{\partial \sigma_{z x}(x, y, z, t)}{\partial x}+\frac{\partial \sigma_{z y}(x, y, z, t)}{\partial y}+\frac{\partial \sigma_{z z}(x, y, z, t)}{\partial z} .\end{array}\right.$

Here $\quad \sigma_{i j}=\frac{E(z)}{2[1+\sigma(z)]}\left[\frac{\partial u_{i}(x, y, z, t)}{\partial x_{j}}+\frac{\partial u_{j}(x, y, z, t)}{\partial x_{i}}-\frac{\delta_{i j}}{3} \frac{\partial u_{k}(x, y, z, t)}{\partial x_{k}}\right]-$ $\chi(z) K(z)\left[T(x, y, z, t)-T_{r}\right]+K(z) \delta_{i j} \frac{\partial u_{k}(x, y, z, t)}{\partial x_{k}}, \rho(z)$ is the density of materials of $\mathrm{H}, \delta_{i j}$ is the Kronecker symbol. After negligible transformation of the last equations one can obtain 


$$
\begin{aligned}
& \rho(z) \frac{\partial^{2} u_{x}(x, y, z, t)}{\partial t^{2}}=\left\{K(z)+\frac{5 E(z)}{6[1+\sigma(z)]}\right\} \frac{\partial^{2} u_{x}(x, y, z, t)}{\partial x^{2}} \\
& +\left\{K(z)-\frac{E(z)}{3[1+\sigma(z)]}\right\} \frac{\partial^{2} u_{y}(x, y, z, t)}{\partial x \partial y} \\
& +\frac{E(z)}{2[1+\sigma(z)]}\left[\frac{\partial^{2} u_{y}(x, y, z, t)}{\partial y^{2}}+\frac{\partial^{2} u_{z}(x, y, z, t)}{\partial z^{2}}\right] \\
& +\left\{K(z)+\frac{E(z)}{3[1+\sigma(z)]}\right\} \frac{\partial^{2} u_{z}(x, y, z, t)}{\partial x \partial z}-K(z) \chi(z) \\
& \times \frac{\partial T(x, y, z, t)}{\partial x} \\
& \rho(z) \frac{\partial^{2} u_{y}(x, y, z, t)}{\partial t^{2}} \\
& =\frac{E(z)}{2[1+\sigma(z)]}\left[\frac{\partial^{2} u_{y}(x, y, z, t)}{\partial x^{2}}+\frac{\partial^{2} u_{x}(x, y, z, t)}{\partial x \partial y}\right] \\
& +\left\{K(z)+\frac{5 E(z)}{12[1+\sigma(z)]}\right\} \times \frac{\partial^{2} u_{y}(x, y, z, t)}{\partial y^{2}} \\
& +\frac{\partial}{\partial z}\left\{\frac{E(z)}{2[1+\sigma(z)]}\left[\frac{\partial u_{y}(x, y, z, t)}{\partial z}+\frac{\partial u_{z}(x, y, z, t)}{\partial y}\right]\right\} \\
& +\left\{K(z)-\frac{5 E(z)}{6[1+\sigma(z)]}\right\} \\
& \times \frac{\partial^{2} u_{x}(x, y, z, t)}{\partial y \partial z}-K(z) \chi(z) \frac{\partial T(x, y, z, t)}{\partial y} \\
& \rho(z) \frac{\partial^{2} u_{z}(x, y, z, t)}{\partial t^{2}} \\
& =\left[\frac{\partial^{2} u_{z}(x, y, z, t)}{\partial x^{2}}+\frac{\partial^{2} u_{z}(x, y, z, t)}{\partial y^{2}}+\frac{\partial^{2} u_{x}(x, y, z, t)}{\partial x \partial z}\right. \\
& \left.+\frac{\partial^{2} u_{x}(x, y, z, t)}{\partial y \partial z}\right] \frac{E(z)}{2[1+\sigma(z)]} \\
& +\frac{\partial}{\partial z}\left\{K(z)\left[\frac{\partial u_{x}(x, y, z, t)}{\partial x}+\frac{\partial u_{y}(x, y, z, t)}{\partial y}+\frac{\partial u_{z}(x, y, z, t)}{\partial z}\right]\right\} \\
& -\frac{\partial T(x, y, z, t)}{\partial z} K(z) \chi(z)+\frac{\partial}{\partial z}\left\{\frac{E(z)}{6[1+\sigma(z)]}\right. \\
& \left.\left[5 \frac{\partial u_{z}(x, y, z, t)}{\partial z}-\frac{\partial u_{x}(x, y, z, t)}{\partial x}-\frac{\partial u_{y}(x, y, z, t)}{\partial y}\right]\right\} \text {. }
\end{aligned}
$$

Initial and boundary conditions for displacement vector could be written as

$$
\begin{aligned}
& \left.\frac{\partial \vec{u}(x, y, z, t)}{\partial x}\right|_{x=0}=\left.\frac{\partial \vec{u}(x, y, z, t)}{\partial x}\right|_{x=L_{x}} ; \\
& \left.\frac{\partial \vec{u}(x, y, z, t)}{\partial y}\right|_{y=0}=\left.\frac{\partial \vec{u}(x, y, z, t)}{\partial y}\right|_{y=L_{y}}=0 ; \\
& \left.\frac{\partial \vec{u}(x, y, z, t)}{\partial z}\right|_{z=0}=\left.\frac{\partial \vec{u}(x, y, z, t)}{\partial z}\right|_{z=L_{z}}=0 ; \\
& \vec{u}(x, y, z, 0)=\vec{u}_{0} ; \quad \vec{u}(x, y, z, \infty)=\vec{u}_{0} .
\end{aligned}
$$

Spatiotemporal distribution of temperature during annealing of dopant could be determined by the second law of Fourier in the following form (Shalimova 1985):

$$
\begin{aligned}
v(T) \frac{\partial T(x, y, z, t)}{\partial t}= & \frac{\partial}{\partial x}\left[\lambda \frac{\partial T(x, y, z, t)}{\partial x}\right] \\
& +\frac{\partial}{\partial y}\left[\lambda \frac{\partial T(x, y, z, t)}{\partial y}\right] \\
& +\frac{\partial}{\partial z}\left[\lambda \frac{\partial T(x, y, z, t)}{\partial z}\right]+p(x, y, z, t)
\end{aligned}
$$

with boundary and initial conditions

$$
\begin{aligned}
& \left.\frac{\partial T(x, y, z, t)}{\partial x}\right|_{x=0}=0,\left.\quad \frac{\partial T(x, y, z, t)}{\partial x}\right|_{x=L_{x}}=0 \\
& \left.\frac{\partial T(x, y, z, t)}{\partial y}\right|_{y=0}=0,\left.\quad \frac{\partial T(x, y, z, t)}{\partial y}\right|_{y=L_{y}}=0, \\
& \left.\frac{\partial T(x, y, z, t)}{\partial z}\right|_{z=0}=0,\left.\quad \frac{\partial T(x, y, z, t)}{\partial z}\right|_{z=L_{z}}=0 \\
& T(x, 0)=f_{T}(x, y, z) .
\end{aligned}
$$

Here $T(x, y, z, t)$ is spatiotemporal distribution of temperature; $\quad v(T)=v_{\text {ass }}\left[1-\theta \exp \left(-T_{d} / T(x, y, z, t)\right)\right] \quad$ is heat capacitance of $\mathrm{H}$ (in the most interest interval of temperature, when temperature $T(x, y, z, t)$ is approximately equal or larger, than Debye temperature $T_{d}$, it could be assumed $v(T) \approx v_{\text {ass }}$ (Shalimova 1985); $\lambda$ is heat conduction coefficient, which value depends on properties of materials of $\mathrm{H}$ and temperature (dependence of heat conduction coefficient on temperature in the most interest interval of values of temperature could be approximated by the following function: $\lambda(x, T)=\lambda_{\text {ass }}(x)\left\{1+\mu\left[T_{d} / T\right.\right.$ $\left.(x, y, z, t)]^{\varphi}\right\}$ (Shalimova 1985); $p(x, y, z, t)$ is volumetric density of heating power of $\mathrm{H} ; \alpha(x, T)=\lambda(x, T) / v(T)$. The framework of this paper attracted an interest in microwave annealing. This type of annealing leads to inhomogenous distribution of temperature (Kuzmichev 1989; Pankratov 2008). The inhomogeneity leads to increasing the sharpness of $p-n$-junction and at the same time leads to increasing the homogeneity of dopant distribution in doped area. In this situation it is practicable to choose such thickness of scinlayer, which approximately is equal to summarized thicknesses of EL and OL.

First of all let us determine spatiotemporal distribution of temperature. To solve the Eq. 5 in pursuance (Pankratov 2005; Pankratov and Spagnolo 2005) we transform the approximation of the heat conduction coefficient $\lambda_{\text {ass }}(x)$ to the following form: $\lambda_{\text {ass }}(x)=\lambda_{\text {oass }}\left[1+\epsilon_{T} g_{T}(x)\right]$, where $\lambda_{\text {Oass }}$ is the average value of the function $\lambda_{\text {ass }}(x)$. Further, we determine solution of the Eq. 5 as the following power series:

$T(x, y, z, t)=\sum_{i=0}^{\infty} \epsilon_{T}^{i} \sum_{j=0}^{\infty} \mu^{j} T_{i j}(x, y, z, t)$.

Substitution of the series Eq. 6 in the Eq. 5 gives us possibility to obtain the system of zero-order 
approximation of temperature $T_{00}(x, t)$ and corrections $T_{i j}(x, t), i \geq 1, j \geq 1$ to the approximation. The equation for zero-order approximation of temperature takes the form

$$
\begin{aligned}
\frac{\partial T_{00}(x, y, z, t)}{\partial t}= & \alpha_{0 \text { ass }}\left[\frac{\partial^{2} T_{00}(x, y, z, t)}{\partial x^{2}}+\frac{\partial^{2} T_{00}(x, y, z, t)}{\partial y^{2}}\right. \\
& \left.+\frac{\partial^{2} T_{00}(x, y, z, t)}{\partial z^{2}}\right]+\frac{p(x, y, z, t)}{v_{\text {ass }}} .
\end{aligned}
$$

Equations for the first- and the second-order corrections are presented in the Appendix. Substitution of the series Eq. 6 into the boundary and initial conditions for temperature leads to the boundary and initial conditions for the all functions $T_{i j}(x, y, z, t)$

$$
\begin{aligned}
& \left.\frac{\partial T_{i j}(x, y, z, t)}{\partial x}\right|_{x=0}=0,\left.\quad \frac{\partial T_{i j}(x, y, z, t)}{\partial x}\right|_{x=L_{x}}=0, \\
& \left.\frac{\partial T_{i j}(x, y, z, t)}{\partial y}\right|_{y=0}=0,\left.\quad \frac{\partial T_{i j}(x, y, z, t)}{\partial x}\right|_{y=L_{y}}=0, \\
& \left.\frac{\partial T_{i j}(x, y, z, t)}{\partial z}\right|_{z=0}=0,\left.\quad \frac{\partial T_{i j}(x, y, z, t)}{\partial x}\right|_{z=L_{z}}=0, \\
& T_{00}(x, 0)=f_{T}(x), \quad T_{i j}(x, 0)=0, \quad i \geq 1, j \geq 1 .
\end{aligned}
$$

Solution of the Eq. 7 with appropriate conditions gives us the following result:

$$
\begin{aligned}
& T_{00}(x, y, z, t)=\frac{F_{0 T}}{L}+\frac{2}{L} \sum_{n=0}^{\infty} F_{n T} C_{n}(x, y, z) e_{n T}(t) \\
& +\frac{1}{L} \int_{0}^{t} \int_{0}^{L_{x}} \int_{0}^{L_{y}} \int_{0}^{L_{z}} \frac{p(u, v, w, t)}{v_{\text {ass }}} \mathrm{d} w \mathrm{~d} v \mathrm{~d} u \mathrm{~d} \tau \\
& +\frac{2}{L} \sum_{n=0}^{\infty} C_{n}(x, y, z) e_{n T}(t) \int_{0}^{t} e_{n T}(-\tau) \int_{0}^{L_{x}} c_{n}(u) \\
& \times \int_{0}^{L_{y}} c_{n}(v) \int_{0}^{L_{z}} c_{n}(w) \frac{p(u, v, w, t)}{v_{\text {ass }}} \mathrm{d} w \mathrm{~d} v \mathrm{~d} u \mathrm{~d} \tau,
\end{aligned}
$$

where $\quad F_{n T}=\int_{0}^{L_{x}} c_{n}(u) \int_{0}^{L_{y}} c_{n}(v) \int_{0}^{L_{z}} c_{n}(w) f_{T}(u, v, w) \mathrm{d} w \mathrm{~d} v$ $\mathrm{d} u, e_{n T}(t)=\exp \left[-\pi^{2} n^{2} \alpha_{0 \text { ass }} t\left(\frac{1}{L_{x}^{2}}+\frac{1}{L_{y}^{2}}+\frac{1}{L_{z}^{2}}\right)\right], c_{n}(\chi)=\cos \left(\frac{\pi n \chi}{L}\right)$, $C_{n}(x, y, z)=c_{n}(x) c_{n}(y) c_{n}(z), L=L_{x} L_{y} L_{z}$. Relations for the first- and the second-order corrections for temperature are presented in the Appendix.

To analyze spatiotemporal distribution of temperature qualitatively and to obtain some quantitative results, the second-order approximations of temperature is good enough approximations (see, for example, Pankratov 2005; Pankratov and Spagnolo 2005). Analytical results give us possibility to obtain and to illustrate demonstrably main

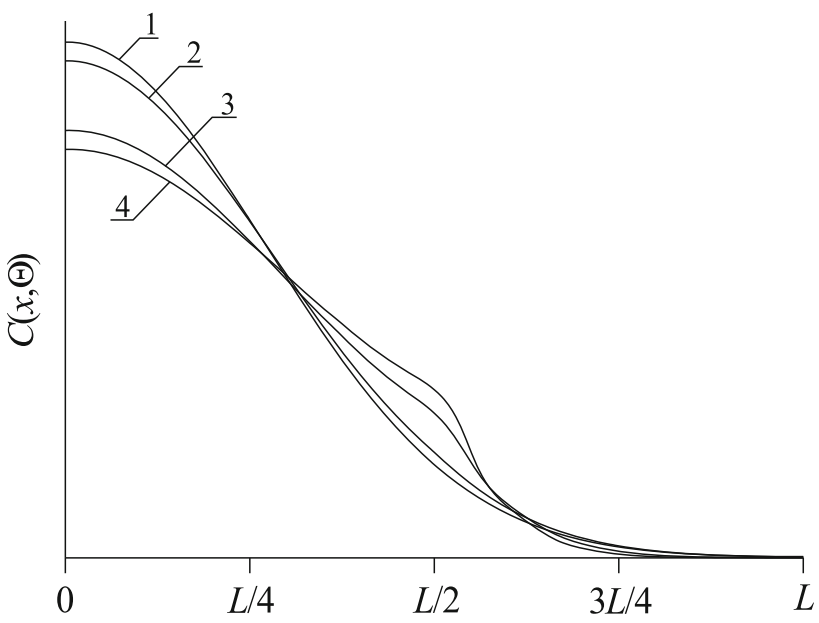

Fig. 2 Distribution of dopant in homogenous sample (curve 1 ) and $\mathrm{H}$ (curves 2-4) for $D_{1}=D_{3}>D_{2}$. Average values of diffusion coefficients in $\mathrm{H}$ are equal to diffusion coefficient in homogenous sample. Curve 2 corresponds to relation $D_{1} / D_{2}=1.2$. Curve 3 corresponds to relation $D_{1} / D_{2}=4.75$. Curve 4 corresponds to relation $D_{1} / D_{2}=11.5$

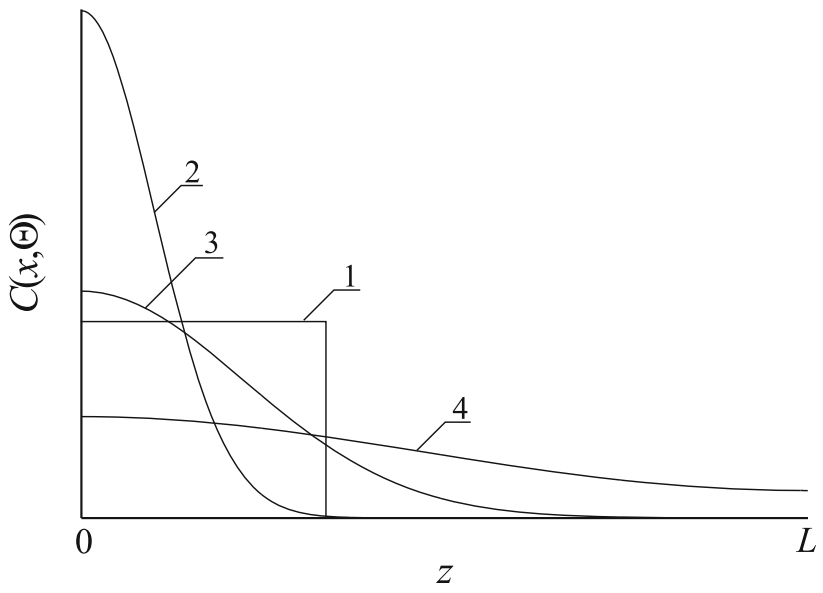

Fig. 3 Curve 1 is idealized step-wise approximation of dopant concentration. Curves 2-4 are real distributions of dopant concentrations for different values of times. The values increase with increasing of the numbers of curves

physical dependencies. To obtain the results with higher exactness numerical approaches have been used. Further, let us estimate values of components of displacement vector. To estimate the values we used method of averaging of function corrections (Pankratov 2008, 2010; Sokolov 1955), because the approach in this case leads to more compact relations. First of all we transform the Eq. 4 to the integro-differential form: 


$$
\begin{aligned}
& u_{x}(x, y, z, t)=u_{x}(x, y, z, t)+\phi_{2}\left\{\frac { 1 } { 6 } \int _ { 0 } ^ { t } \int _ { 0 } ^ { z } \left[5 \frac{\partial^{2} u_{x}(x, y, w, \tau)}{\partial x^{2}}\right.\right. \\
& u_{y}(x, y, z, t)=u_{y}(x, y, z, t) \\
& +\phi_{2}\left\{\frac{1}{2} \int_{0}^{t}(t-\tau) \int_{0}^{z}\left[5 \frac{\partial^{2} u_{y}(x, y, w, \tau)}{\partial x^{2}}+\frac{\partial^{2} u_{x}(x, y, w, \tau)}{\partial x \partial y}\right]\right. \\
& \times \frac{E(w) \mathrm{dw}}{1+\sigma(w)} \mathrm{d} \tau-\frac{t}{2} \int_{0}^{\infty} \int_{0}^{z}\left[5 \frac{\partial^{2} u_{y}(x, y, w, \tau)}{\partial x^{2}}+\frac{\partial^{2} u_{x}(x, y, w, \tau)}{\partial x \partial y}\right] \\
& \times \frac{E(w) \mathrm{dw}}{1+\sigma(w)} \mathrm{d} \tau+\frac{1}{6} \int_{0}^{t}(t-\tau) \int_{0}^{z}\left[5 \frac{\partial^{2} u_{y}(x, y, w, \tau)}{\partial y^{2}}\right. \\
& \left.+\frac{\partial^{2} u_{x}(x, y, w, \tau)}{\partial x \partial y}+\frac{\partial^{2} u_{z}(x, y, w, \tau)}{\partial y \partial w}\right] \frac{E(w) \mathrm{d} w}{1+\sigma(w)} \mathrm{d} \tau \\
& -\frac{t}{6} \int_{0}^{\infty} \int_{0}^{z}\left[5 \frac{\partial^{2} u_{y}(x, y, w, \tau)}{\partial y^{2}}+\frac{\partial^{2} u_{x}(x, y, w, \tau)}{\partial x \partial y}\right. \\
& \left.+\frac{\partial^{2} u_{z}(x, y, w, \tau)}{\partial y \partial w}\right] \frac{E(w) \mathrm{d} w}{1+\sigma(w)} \mathrm{d} \tau+\int_{0}^{t} \int_{0}^{z}\left[5 \frac{\partial^{2} u_{y}(x, y, w, \tau)}{\partial y^{2}}\right. \\
& \left.+\frac{\partial^{2} u_{y}(x, y, w, \tau)}{\partial x \partial y}+\frac{\partial^{2} u_{y}(x, y, w, \tau)}{\partial y \partial w}\right] \\
& \times K(w) \mathrm{d} w(t-\tau) \mathrm{d} \tau-t \int_{0}^{\infty} \int_{0}^{z}\left[5 \frac{\partial^{2} u_{y}(x, y, w, \tau)}{\partial y^{2}}\right. \\
& \left.+\frac{\partial^{2} u_{y}(x, y, w, \tau)}{\partial x \partial y}+\frac{\partial^{2} u_{y}(x, y, w, \tau)}{\partial y \partial w}\right] K(w) \mathrm{d} w \mathrm{~d} \tau \\
& +\frac{E(z)}{2[1+\sigma(z)]} \int_{0}^{t}(t-\tau)\left[\frac{\partial u_{y}(x, y, z, \tau)}{\partial z}+\frac{\partial u_{z}(x, y, z, \tau)}{\partial y}\right] \mathrm{d} \tau \\
& -\frac{t E(z)}{2[1+\sigma(z)]} \int_{0}^{\infty}\left[\frac{\partial u_{y}(x, y, z, \tau)}{\partial z}+\frac{\partial u_{z}(x, y, z, \tau)}{\partial y}\right] \mathrm{d} \tau \\
& -\int_{0}^{t}(t-\tau) \int_{0}^{z} K(w) \chi(w) \frac{\partial T(x, y, w, \tau)}{\partial y} \mathrm{~d} w \mathrm{~d} \tau \\
& +t \int_{0}^{t} \int_{0}^{z} K(w) \frac{\partial T(x, y, w, \tau)}{\partial y} \times \chi(w) \mathrm{d} w \mathrm{~d} \tau \\
& -\int_{0}^{t}(t-\tau) \int_{0}^{z} \rho(w) \frac{\partial^{2} u_{y}(x, y, w, \tau)}{\partial \tau^{2}} \mathrm{~d} w \mathrm{~d} \tau . \\
& +t \int_{0}^{\infty} \int_{0}^{z} \rho(w) \frac{\partial^{2} u_{y}(x, y, w, \tau)}{\partial \tau^{2}} \mathrm{~d} w \mathrm{~d} \tau \\
& \left.-\Phi_{y 1}(x, y, z, t)\right\}
\end{aligned}
$$




$$
\begin{aligned}
& u_{z}(x, y, z, t)=u_{z}(x, y, z, t)+\phi_{2}\left\{\frac { 1 } { 2 } \int _ { 0 } ^ { t } \int _ { 0 } ^ { z } \left[5 \frac{\partial^{2} u_{z}(x, y, w, \tau)}{\partial x^{2}}\right.\right. \\
& \left.+\frac{\partial^{2} u_{x}(x, y, w, \tau)}{\partial x \partial w}\right] \frac{E(w) \mathrm{d} w}{1+\sigma(w)}(t-\tau) \mathrm{d} \tau \\
& -\frac{t}{2} \int_{0}^{\infty} \int_{0}^{z}\left[5 \frac{\partial^{2} u_{z}(x, y, w, \tau)}{\partial x^{2}}+\frac{\partial^{2} u_{x}(x, y, w, \tau)}{\partial x \partial w}\right] \\
& \times \frac{E(w) \mathrm{d} w}{1+\sigma(w)} \mathrm{d} \tau+\frac{1}{2} \int_{0}^{t} \int_{0}^{z} \frac{E(w)}{1+\sigma(w)} \\
& \times\left[5 \frac{\partial^{2} u_{z}(x, y, w, \tau)}{\partial y^{2}}+\frac{\partial^{2} u_{y}(x, y, w, \tau)}{\partial y \partial w}\right] \mathrm{d} w \mathrm{~d} \tau \\
& -\int_{0}^{\infty} \int_{0}^{z}\left[5 \frac{\partial^{2} u_{z}(x, y, w, \tau)}{\partial y^{2}}+\frac{\partial^{2} u_{y}(x, y, w, \tau)}{\partial y \partial w}\right] \\
& \times \frac{t}{2} \frac{E(w) \mathrm{d} w}{1+\sigma(w)} \mathrm{d} \tau+\int_{0}^{t} \frac{E(z)(t-\tau)}{6[1+\sigma(z)]}\left[5 \frac{\partial u_{z}(x, y, z, \tau)}{\partial z}\right. \\
& \left.-\frac{\partial u_{x}(x, y, z, \tau)}{\partial x}-\frac{\partial u_{y}(x, y, z, \tau)}{\partial y}\right] \mathrm{d} \tau \\
& -\frac{t E(z)}{6[1+\sigma(z)]} \int_{0}^{\infty}\left[5 \frac{\partial u_{z}(x, y, z, \tau)}{\partial z}-\frac{\partial u_{x}(x, y, z, \tau)}{\partial x}\right. \\
& \left.-\frac{\partial u_{y}(x, y, z, \tau)}{\partial y}\right] \mathrm{d} \tau+K(z) \int_{0}^{t}(t-\tau) \\
& \times\left[\frac{\partial u_{x}(x, y, z, \tau)}{\partial x}+\frac{\partial u_{y}(x, y, z, \tau)}{\partial y}+\frac{\partial u_{z}(x, y, z, \tau)}{\partial z}\right] \mathrm{d} \tau \\
& -t K(z) \int_{0}^{\infty}\left[\frac{\partial u_{x}(x, y, z, \tau)}{\partial x}+\frac{\partial u_{y}(x, y, z, \tau)}{\partial y}\right. \\
& \left.+\frac{\partial u_{z}(x, y, z, \tau)}{\partial z}\right] \mathrm{d} \tau+t \int_{0}^{\infty} \int_{0}^{z} K(w) \chi(w) \frac{\partial T(x, y, w, \tau)}{\partial w} \mathrm{~d} w \mathrm{~d} \tau \\
& \left.-\int_{0}^{t} \int_{0}^{z} K(w) \chi(w) \frac{\partial T(x, y, w, \tau)}{\partial w} \mathrm{~d} w\right\} .
\end{aligned}
$$

Here $E_{0}$ is the average value of Young modulus, $\phi_{2}=L / \Theta^{2} E_{0}, \Phi_{\beta i}=\int_{0}^{z} \rho(w)\left[u_{\beta}(x, y, w, t)\right]^{i} \mathrm{~d} w$.

The first-order approximations of components of displacement vector were obtained by replacement of the functions $u_{\beta}(x, y, z, t)$ on their average values in right sides of Eqs. 9, i.e. $u_{\beta}(x, y, z, t) \rightarrow \alpha_{u \beta 1}, \beta=x, y, z$. The average values could be calculated by the following relation:

$\alpha_{u \beta 1}=\frac{M_{\beta 1}}{4 L \Theta}$,

where $\quad M_{\beta i}=\int_{0}^{\Theta} \int_{-L_{x}}^{L_{x}} \int_{-L_{y}}^{L_{y}} \int_{0}^{L_{z}} u_{\beta i}(x, y, z, t) \mathrm{d} z \mathrm{~d} y \mathrm{~d} x \mathrm{~d} t$. For concrete values of $\beta$ one can obtain

$$
\begin{aligned}
u_{x 1}(x, y, z, t)= & \alpha_{u x 1}+\left[t \int_{0}^{\infty} \int_{0}^{z} K(w) \chi(w) \frac{\partial T(x, y, w, \tau)}{\partial x} \mathrm{~d} w \mathrm{~d} \tau\right. \\
& \left.-\int_{0}^{t}(t-\tau) \int_{0}^{z} K(w) \chi(w) \frac{\partial T(x, y, w, \tau)}{\partial x} \mathrm{~d} w \mathrm{~d} \tau\right] \\
u_{y 1}(x, y, z, t)= & \alpha_{u y 1}+\left[t \int_{0}^{\infty} \int_{0}^{z} K(w) \chi(w) \frac{\partial T(x, y, w, \tau)}{\partial y} \mathrm{~d} w \mathrm{~d} \tau\right. \\
& \left.-\int_{0}^{t}(t-\tau) \int_{0}^{z} K(w) \chi(w) \frac{\partial T(x, y, w, \tau)}{\partial y} \mathrm{~d} w \mathrm{~d} \tau\right] \\
u_{z 1}(x, y, z, t)= & \alpha_{u z 1}+\left[\int_{0}^{\infty} \int_{0}^{t} K(w) \chi(w) \frac{\partial T(x, y, w, \tau)}{\partial w} \mathrm{~d} w \mathrm{~d} \tau\right. \\
& \left.-\int_{0}^{t}(t-\tau) \int_{0}^{z} K(w) \chi(w) \frac{\partial T(x, y, w, \tau)}{\partial w} \mathrm{~d} w \mathrm{~d} \tau\right] .
\end{aligned}
$$

Substitution of the last relations into Eq. 9 gives us possibility to determine the parameters $\alpha_{u \beta 1}$. The parameters after appropriate calculations could be written as

$$
\begin{aligned}
& \alpha_{u x 1}=\Theta \frac{L_{z}\left[\Xi_{x 0}(\infty)-\Xi_{x 2}(\Theta)\right]}{8 L^{3} \Lambda} ; \\
& \alpha_{u y 1}=\Theta \frac{L_{z}\left[\Xi_{y 0}(\infty)-\Xi_{y 2}(\Theta)\right]}{8 L^{3} \Lambda} ; \\
& \alpha_{u z 1}=\Theta \frac{L_{z}\left[\Xi_{z 0}(\infty)-\Xi_{z 2}(\Theta)\right]}{8 L^{3} \Lambda} ;
\end{aligned}
$$

where $\Xi_{\beta i}=\int_{0}^{\Theta}\left(1+\frac{t}{\Theta}\right)^{i} \int_{-L_{x}}^{L_{x}} \int_{-L_{y}}^{L_{y}} \int_{0}^{L_{z}}\left(L_{z}-z\right) K(z) \chi(z) \frac{\partial T(x, y, z, t)}{\partial \beta}$ $\mathrm{d} z \mathrm{~d} y \mathrm{~d} x \mathrm{~d} t, \Lambda=\int_{0}^{L_{z}}\left(L_{z}-z\right) \rho(z) \mathrm{d} z$.

The second-order approximations of components of displacement vector were obtained by replacement of the functions $u_{\beta}(x, y, z, t)$ on the following sums $\alpha_{u} \beta_{2}+$ $u_{\beta 1}(x, y, z, t)$ in right sides of Eqs. 8. Here $\alpha_{u \beta 2}=\left(M_{u \beta 2}-\right.$ $\left.M_{u \beta 1}\right) / 4 L^{3} \Theta$. The results of calculation of the second-order approximations and parameters $\alpha_{u \beta 2}$ are bulky and, therefore, were presented in the Appendix.

Further, we solved Eq. 1. To obtain the solution let us transform dopant diffusion coefficients $D_{L}(z, T)$ and $D_{S L}(z, T)$ in the following form: $D_{L}(z, T)=D_{0 L}[1+$ $\left.\epsilon_{L} g_{L}(z, T)\right]$ and $D_{S L}(z, T)=D_{0 S L}\left[1+\epsilon_{S} g_{S}(z, T)\right]$. Also we introduce the dimensionless parameter $\omega=D_{O L} / D_{0} \quad S L$. In this situation Eq. 1 takes the form

$$
\begin{aligned}
& \frac{\partial C(x, y, z, t)}{\partial t}=D_{0 L} \frac{\partial}{\partial x}\left\{\left[1+\epsilon_{L} g_{L}(z, T)\right] \frac{\partial C(x, y, z, t)}{\partial x}\right\} \\
& +D_{0 L} \frac{\partial}{\partial y}\left\{\left[1+\epsilon_{L} g_{L}(z, T)\right] \frac{\partial C(x, y, z, t)}{\partial y}\right\} \\
& +D_{0 L} \frac{\partial}{\partial z}\left\{\left[1+\epsilon_{L} g_{L}(z, T)\right] \frac{\partial C(x, y, z, t)}{\partial z}\right\}
\end{aligned}
$$




$$
\begin{aligned}
& +\Omega D_{0 S L} \frac{\partial}{\partial x}\left\{\frac{\left[1+\epsilon_{S} g_{S}(z, T)\right]}{k T} \nabla_{S} \mu(x, y, z, t)\right. \\
& \left.\times \int_{0}^{L_{z}} C(x, y, W, t) d W\right\}+\Omega D_{0 S L} \frac{\partial}{\partial y} \\
& \times\left\{\frac{\left[1+\epsilon_{S} g_{S}(\mathrm{z}, \mathrm{T})\right]}{\mathrm{kT}} \nabla_{\mathrm{S}} \mu(\mathrm{x}, \mathrm{y}, \mathrm{z}, \mathrm{t})\right. \\
& \left.\times \int_{0}^{L_{z}} C(x, y, W, t) d W\right\} .
\end{aligned}
$$

Let us to solve Eq. 1 as the power series

$$
C(x, y, z, t)=\sum_{i=0}^{\infty} \epsilon_{L}^{i} \sum_{j=0}^{\infty} \xi^{j} \sum_{k=0}^{\infty} \omega^{k} C_{i j k}(x, y, z, t) .
$$

Substitution of the series Eq. 12 in the Eq. 11 gives us possibility to obtain equations for zeroth-order approximation of concentration of dopant $C_{000}(x, y, z, t)$ and corrections to the approximations $C_{i j k}(x, y, z, t)(i \geq 1$, $j \geq 1$ ). The equation for zeroth-order approximation could be written as

$$
\begin{aligned}
\frac{\partial C_{000}(x, y, z, t)}{\partial t}= & D_{0 L} \frac{\partial^{2} C_{000}(x, y, z, t)}{\partial x^{2}} \\
& +D_{0 L} \frac{\partial^{2} C_{000}(x, y, z, t)}{\partial y^{2}} \\
& +D_{0 L} \frac{\partial^{2} C_{000}(x, y, z, t)}{\partial z^{2}} .
\end{aligned}
$$

Equations for the first- and the second-order corrections to zeroth-order approximation of concentration of dopant are presented in the Appendix.

Substitution of the series (12) into appropriate boundary and initial conditions gives us possibility to obtain boundary and initial conditions for the functions $C_{i j k}(x, y, z, t)$ in the following form:

$$
\begin{aligned}
& \left.\frac{\partial C_{i j k}(x, y, z, t)}{\partial x}\right|_{x=0}=\left.\frac{\partial C_{i j k}(x, y, z, t)}{\partial x}\right|_{x=L_{x}}=0 \\
& \left.\frac{\partial C_{i j k}(x, y, z, t)}{\partial y}\right|_{y=0}=\left.\frac{\partial C_{i j k}(x, y, z, t)}{\partial y}\right|_{y=L_{y}}=0 \\
& \begin{aligned}
&\left.\frac{\partial C_{i j k}(x, y, z, t)}{\partial z}\right|_{z=0}=\left.\frac{\partial C_{i j k}(x, y, z, t)}{\partial z}\right|_{z=L_{z}}=0 \\
& C_{000}(x, y, z, 0)=f_{c}(x, y, z), C_{i j k}(x, y, z, 0) \\
&=0, i \geq 1, j \geq 1, k \geq 1 .
\end{aligned}
\end{aligned}
$$

Solutions of equations for the functions $C_{i j k}(x, y$, $z, t$ ) could be written as

$$
\begin{aligned}
C_{000}(x, y, z, t)= & \frac{F_{0 C}}{L_{x} L_{y} L_{z}} \\
& +\frac{2}{L_{x} L_{y} L_{z}} \sum_{n=0}^{\infty} F_{n C} c_{n}(x) c_{n}(y) c_{n}(z) e_{n C}(t) .
\end{aligned}
$$

Here $F_{n C}=\int_{0}^{L_{x}} c_{n}(u) \int_{0}^{L_{x}} c_{n}(v) \int_{0}^{L_{x}} c_{n}(w) f_{C}(u, v, w) \mathrm{d} w \mathrm{~d} v \mathrm{~d} u$, $e_{n C}(t)=\exp \left[-\pi^{2} n^{2} D_{0 L} t\left(\frac{1}{L_{x}^{2}}+\frac{1}{L_{y}^{2}}+\frac{1}{L_{y}^{2}}\right)\right]$. Other solutions are presented in Appendix.

Analysis of spatiotemporal distribution of dopant concentration has been done analytically using the secondorder approximation of dopant concentration. Further, the distribution has been amended numerically.

\section{Discussion}

In this paragraph we analyzed spatiotemporal distribution of dopant in considered $\mathrm{H}$ (see Fig. 1) during annealing. It has been recently shown (see, for example, Pankratov 2005, 2008, 2010; Pankratov and Spagnolo 2005) that achievement of the interfaces between EL and S gives us possibility to increase sharpness of $p-n$-junction and homogeneity of dopant distribution in enriched area. Fig. 2 illustrates dopant distributions in homogenous sample and in $\mathrm{H}$ after annealing with equal continuance. Decreasing of annealing time leads to decreasing of homogeneity of dopant distribution. Increasing of annealing time leads to increasing of homogeneity of dopant distribution and to decreasing of sharpness of $p-n$-junction. Both effects have been illustrated by Fig. 3 .

It is known that in any $\mathrm{H}$ mechanical stress exists due to displacement lattice spacing. We have also shown that the mechanical stress did not lead to qualitatively new distributions of dopant in $z$-direction. However, mechanical stress leads to increasing density of $p-n$-junctions, which comprise of IC (see Pankratov 2010). The increasing has been achieved due to deceleration of lateral diffusion. The deceleration could be obtained for negative value of mismatch

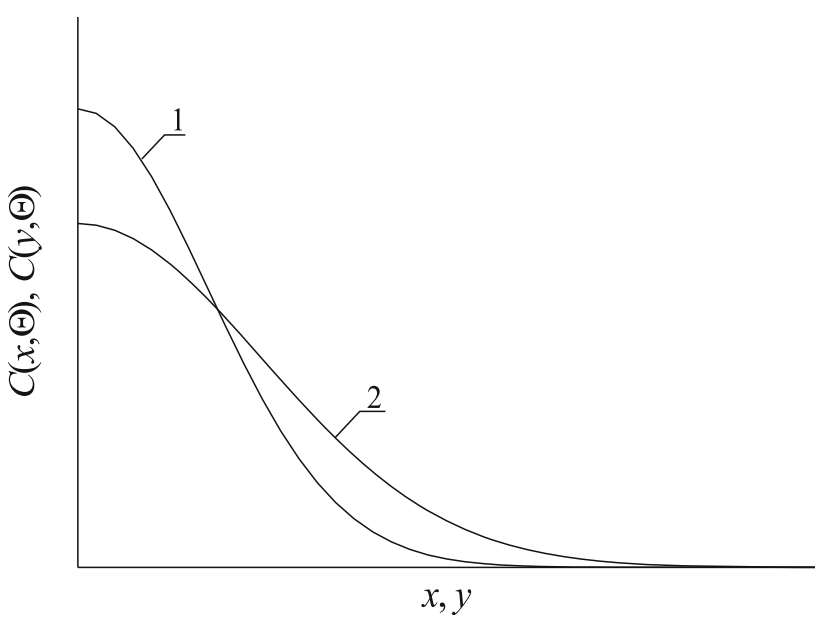

Fig. 4 Distribution of dopant in $\mathrm{H}$ in $x$ - and $y$-directions. Curve 1 corresponds to negative value of mismatch strain $\epsilon_{0}$ between EL and S. Curve 2 corresponds to positive value of mismatch strain $\epsilon_{0}$ between EL and $\mathrm{S}$ 
strain $\epsilon_{0}$ between EL and S and positive value of mismatch strain $\epsilon_{0}$ between OL and EL. The dependence is illustrated in Fig. 4. In this paper the result has been generalised for larger number of epitaxial layers. It has been obtained that using overlayer gives us possibility to increase density of $p-$ $n$-junctions near $z=0$ in comparison with free surface $z=0$. After the increasing the overlayer could be etched, if necessary. It has been also obtained that using only mechanical stress did not lead to maximal increasing of density of $p-n$ - junctions. To obtain higher density, $\mathrm{H}$ with mesh EL could be used (see Pankratov 2009).

\section{Conclusion}

In this paper we analyzed the influence of mechanical stress in heterostructure on depth of $p-n$-junctions and their density in integrated circuits. Some conditions have been formulated to increase density of $p-n$-junctions in integrated circuits.

Acknowledgments This work has been supported by grant of President of Russia (project MK-548.2010.2).

Open Access This article is distributed under the terms of the Creative Commons Attribution License which permits any use, distribution and reproduction in any medium, provided the original author(s) and source are credited.

\section{Appendix}

Equations for the first- and the second-order corrections could be written as

$$
\begin{aligned}
& \frac{\partial T_{i 0}(x, y, z, t)}{\partial t}=\alpha_{0 \text { ass }}\left[\frac{\partial^{2} T_{i 0}(x, y, z, t)}{\partial x^{2}}+\frac{\partial^{2} T_{i 0}(x, y, z, t)}{\partial y^{2}}\right. \\
& \left.+\frac{\partial^{2} T_{i 0}(x, y, z, t)}{\partial z^{2}}\right]+\alpha_{0 \text { ass }}\left\{g_{T}(z) \frac{\partial^{2} T_{i-10}(x, y, z, t)}{\partial x^{2}}\right. \\
& \left.+g_{T}(z) \frac{\partial^{2} T_{i-10}(x, y, z, t)}{\partial y^{2}}+\frac{\partial}{\partial z}\left[g_{T}(z) \frac{\partial T_{i-10}(x, y, z, t)}{\partial z}\right]\right\} \\
& i \geq 1 \\
& \frac{\partial T_{01}(x, y, z, t)}{\partial t}=\alpha_{0 \text { ass }}\left[\frac{\partial^{2} T_{01}(x, y, z, t)}{\partial x^{2}}+\frac{\partial^{2} T_{01}(x, y, z, t)}{\partial y^{2}}\right. \\
& \left.\quad+\frac{\partial^{2} T_{01}(x, y, z, t)}{\partial z^{2}}\right]+\frac{\alpha_{0 a s s} T_{d}^{\varphi}}{T_{00}^{\varphi}(x, y, z, t)} \\
& \quad \times\left[\frac{\partial^{2} T_{00}(x, y, z, t)}{\partial x^{2}}+\frac{\partial^{2} T_{00}(x, y, z, t)}{\partial y^{2}}+\frac{\partial^{2} T_{00}(x, y, z, t)}{\partial z^{2}}\right] \\
& \left.\quad+\left[\frac{\partial T_{00}(x, y, z, t)}{\partial y}\right]^{2}+\left[\frac{\partial T_{00}(x, y, z, t)}{\partial z}\right]^{2}\right\}
\end{aligned}
$$

$$
\begin{aligned}
& \frac{\partial T_{02}(x, y, z, t)}{\partial t}=\alpha_{0 \text { ass }}\left[\frac{\partial^{2} T_{02}(x, y, z, t)}{\partial x^{2}}+\frac{\partial^{2} T_{02}(x, y, z, t)}{\partial y^{2}}\right. \\
& \left.+\frac{\partial^{2} T_{02}(x, y, z, t)}{\partial z^{2}}\right]\left[1+\frac{\alpha_{0 a s s} T_{d}^{\varphi}}{T_{00}^{\varphi}(x, y, z, t)}\right] \\
& -\left[\frac{\partial T_{00}(x, y, z, t)}{\partial x} \frac{\partial T_{01}(x, y, z, t)}{\partial x}+\frac{\partial T_{00}(x, y, z, t)}{\partial y}\right. \\
& \left.\times \frac{\partial \mathrm{T}_{01}(\mathrm{x}, \mathrm{y}, \mathrm{z}, \mathrm{t})}{\partial \mathrm{y}}+\frac{\partial \mathrm{T}_{00}(\mathrm{x}, \mathrm{y}, \mathrm{z}, \mathrm{t})}{\partial \mathrm{z}} \frac{\partial \mathrm{T}_{01}(\mathrm{x}, \mathrm{y}, \mathrm{z}, \mathrm{t})}{\partial \mathrm{z}}\right] \\
& \times \frac{\varphi \alpha_{0 \text { ass }} T_{d}^{\varphi}}{T_{00}^{\varphi+1}(x, y, z, t)} \\
& \frac{\partial T_{11}(x, y, z, t)}{\partial t}=\alpha_{0 \text { ass }}\left[\frac{\partial^{2} T_{11}(x, y, z, t)}{\partial x^{2}}+\frac{\partial^{2} T_{11}(x, y, z, t)}{\partial y^{2}}\right. \\
& \left.+\frac{\partial^{2} T_{11}(x, y, z, t)}{\partial z^{2}}\right]+\alpha_{0 \text { ass }} \frac{T_{01}(x, y, z, t)}{T_{00}(x, y, z, t)} \\
& \times\left\{g_{T}(z) \frac{\partial^{2} T_{00}(x, y, z, t)}{\partial x^{2}}+g_{T}(z) \frac{\partial^{2} T_{00}(x, y, z, t)}{\partial y^{2}}\right. \\
& \left.+\frac{\partial}{\partial z}\left[g_{T}(z) \frac{\partial T_{00}(x, y, z, t)}{\partial z}\right]\right\}+\frac{\partial^{2} T_{01}(x, y, z, t)}{\partial x^{2}} \\
& \times \alpha_{0 \text { ass }}+\alpha_{0 \text { ass }} \frac{\partial^{2} T_{01}(x, y, z, t)}{\partial y^{2}}+\alpha_{0 \text { ass }} \frac{\partial^{2} T_{01}(x, y, z, t)}{\partial z^{2}} \\
& +\alpha_{0 \text { ass }}\left\{g_{T}(z) \frac{\partial^{2} T_{01}(x, y, z, t)}{\partial x^{2}}+g_{T}(z) \times \frac{\partial^{2} T_{01}(x, y, z, t)}{\partial y^{2}}\right. \\
& \left.+\frac{\partial}{\partial z}\left[g_{T}(z) \frac{\partial T_{01}(x, y, z, t)}{\partial z}\right]\right\}+\left[\frac{\partial^{2} T_{00}(x, y, z, t)}{\partial x^{2}}\right. \\
& \left.+\frac{\partial^{2} T_{00}(x, y, z, t)}{\partial y^{2}}+\frac{\partial^{2} T_{00}(x, y, z, t)}{\partial z^{2}}\right] \\
& \times \alpha_{0 \text { ass }} \frac{T_{d}^{\varphi} T_{10}(x, y, z, t)}{T_{00}^{\varphi+1}(x, y, z, t)}+\frac{\alpha_{0 \text { ass }} T_{d}^{\varphi}}{T_{00}^{\varphi}(x, y, z, t)}\left[\frac{\partial^{2} T_{10}(x, y, z, t)}{\partial x^{2}}\right. \\
& \left.+\frac{\partial^{2} T_{10}(x, y, z, t)}{\partial y^{2}}+\frac{\partial^{2} T_{10}(x, y, z, t)}{\partial z^{2}}\right] \\
& +\frac{\alpha_{0 \text { ass }} T_{d}^{\varphi}}{T_{00}^{\varphi}(x, y, z, t)}\left\{g_{T}(z) \frac{\partial^{2} T_{00}(x, y, z, t)}{\partial x^{2}}\right. \\
& \left.+g_{T}(z) \frac{\partial^{2} T_{00}(x, y, z, t)}{\partial y}+\frac{\partial}{\partial z}\left[g_{T}(z) \frac{\partial T_{00}(x, y, z, t)}{\partial z}\right]\right\} \\
& -\left[\frac{\partial T_{00}(x, y, z, t)}{\partial x} \frac{\partial T_{10}(x, y, z, t)}{\partial x}+\frac{\partial T_{00}(x, y, z, t)}{\partial y}\right. \\
& \left.\times \frac{\partial \mathrm{T}_{10}(\mathrm{x}, \mathrm{y}, \mathrm{z}, \mathrm{t})}{\partial \mathrm{y}}+\frac{\partial \mathrm{T}_{00}(\mathrm{x}, \mathrm{y}, \mathrm{z}, \mathrm{t})}{\partial \mathrm{z}} \frac{\partial \mathrm{T}_{10}(\mathrm{x}, \mathrm{y}, \mathrm{z}, \mathrm{t})}{\partial \mathrm{z}}\right] \\
& \times \frac{\varphi \alpha_{0 \mathrm{ass}} T_{d}^{\varphi}}{T_{00}^{\varphi+1}(x, y, z, t)}-\varphi \frac{g_{T}(x) \alpha_{0 \mathrm{ass}} T_{d}^{\varphi}}{T_{00}^{\varphi+1}(x, y, z, t)}\left\{\left[\frac{\partial T_{00}(x, y, z, t)}{\partial x}\right]^{2}\right. \\
& \left.+\left[\frac{\partial T_{00}(x, y, z, t)}{\partial y}\right]^{2}+\left[\frac{\partial T_{00}(x, y, z, t)}{\partial z}\right]^{2}\right\} \text {. }
\end{aligned}
$$

Relations for the first- and the second-order corrections for temperature take the form 


$$
\begin{aligned}
& T_{i 0}(x, y, z, t)=\frac{2 \pi \alpha_{0 a s s}}{L_{x}^{2} L_{y} L_{z}} \sum_{n=0}^{\infty} n C_{n}(x, y, z) e_{n T}(t) \int_{0}^{t} e_{n T}(-\tau) \\
& \times \int_{0}^{\mathrm{L}_{\mathrm{x}}} \mathrm{s}_{\mathrm{n}}(\mathrm{u}) \int_{0}^{\mathrm{L}_{\mathrm{y}}} \mathrm{c}_{\mathrm{n}}(\mathrm{v}) \int_{0}^{\mathrm{L}_{z}} \mathrm{~g}_{\mathrm{T}}(\mathrm{w}) \frac{\partial \mathrm{T}_{\mathrm{i}-10}(\mathrm{u}, \mathrm{v}, \mathrm{w}, \tau)}{\partial \mathrm{u}} \\
& \times c_{n}(w) \mathrm{d} w \mathrm{~d} v \mathrm{~d} u \mathrm{~d} \tau+\frac{2 \pi \alpha_{0 \mathrm{ass}}}{L_{x} L_{y}^{2} L_{z}} \sum_{n=0}^{\infty} n C_{n}(x, y, z) e_{n T}(t) \\
& \times \int_{0}^{\mathrm{t}} \mathrm{e}_{\mathrm{nT}}(-\tau) \int_{0}^{\mathrm{L}_{\mathrm{x}}} \mathrm{c}_{\mathrm{n}}(\mathrm{u}) \int_{0}^{\mathrm{L}_{\mathrm{y}}} \mathrm{s}_{\mathrm{n}}(\mathrm{v}) \int_{0}^{\mathrm{L}_{z}} \mathrm{c}_{\mathrm{n}}(\mathrm{w}) \mathrm{g}_{\mathrm{T}}(\mathrm{w}) \\
& \times \frac{\partial T_{i-10}(u, v, w, \tau)}{\partial v} \mathrm{~d} w \mathrm{~d} v \mathrm{~d} u \mathrm{~d} \tau+\frac{2 \pi \alpha_{0 a s s}}{L_{x} L_{y} L_{z}^{2}} \\
& \quad \times \sum_{n=0}^{\infty} n C_{n}(x, y, z) e_{n T}(t) \int_{0}^{t} e_{n T}(-\tau) \int_{0}^{L_{x}} c_{n}(u) \int_{0}^{L_{y}} c_{n}(v) \\
& \times \int_{0}^{L_{z}} s_{n}(w) g_{T}(w) \frac{\partial T_{i-10}(u, v, w, \tau)}{\partial w} \mathrm{~d} w \mathrm{~d} v \mathrm{~d} u \mathrm{~d} \tau, i \geq 1,
\end{aligned}
$$$$
T_{02}(x, y, z, t)=T_{d}^{\varphi} \frac{2 \pi \alpha_{0 \text { ass }}}{L_{x}^{2} L_{y} L_{z}} \sum_{n=0}^{\infty} n C_{n}(x, y, z) e_{n T}(t) \int_{0}^{t} e_{n T}(-\tau)
$$$$
\times \int_{0}^{L_{x}} s_{n}(u) \int_{0}^{L_{y}} c_{n}(v) \int_{0}^{L_{z}} \frac{\partial^{2} T_{01}(u, v, w, \tau)}{\partial u^{2}}
$$$$
\times c_{n}(w) \frac{\mathrm{dwdvdud} \tau}{T_{00}^{\varphi}(u, v, w, \tau)}+T_{d}^{\varphi} \frac{2 \pi \alpha_{0 \text { ass }}}{L_{x} L_{y}^{2} L_{z}} \sum_{n=0}^{\infty} n C_{n}(x, y, z) e_{n T}(t)
$$$$
\times \int_{0}^{t} e_{n T}(-\tau) \int_{0}^{L_{x}} c_{n}(u) \int_{0}^{L_{y}} s_{n}(v) \int_{0}^{L_{z}} c_{n}(w)
$$$$
\times \frac{\partial^{2} T_{01}(u, v, w, \tau)}{\partial v^{2}} \frac{\mathrm{dwdvdud} \tau}{T_{00}^{\varphi}(u, v, w, \tau)}+T_{d}^{\varphi} \frac{2 \pi \alpha_{0 \text { ass }}}{L_{x} L_{y} L_{z}^{2}}
$$$$
\times \sum_{n=0}^{\infty} n C_{n}(x, y, z) e_{n T}(t) \int_{0}^{t} e_{n T}(-\tau) \int_{0}^{L_{x}} c_{n}(u) \int_{0}^{L_{y}} c_{n}(v)
$$$$
\times \int_{0}^{L_{z}} s_{n}(w) \frac{\partial^{2} T_{01}(u, v, w, \tau)}{\partial w^{2}} \frac{\mathrm{dwdvdud} \tau}{T_{00}^{\varphi}(u, v, w, \tau)}
$$$$
-\varphi T_{d}^{\varphi} \frac{2 \pi \alpha_{0 \mathrm{ass}}}{L_{x}^{2} L_{y} L_{z}} \sum_{n=0}^{\infty} C_{n}(x, y, z) e_{n T}(t) \int_{0}^{t} e_{n T}(-\tau) \int_{0}^{L_{x}} s_{n}(u)
$$$$
\times \int_{0}^{L_{y}} c_{n}(v) \int_{0}^{L_{z}} c_{n}(w) \frac{\partial T_{00}(u, v, w, \tau)}{\partial u} \frac{\partial T_{01}(u, v, w, \tau)}{L^{2}}
$$$$
\times \int_{0}^{t} e_{n T}(-\tau) \int_{0}^{L_{x}} s_{n}(u) \int_{0}^{L_{y}} c_{n}(v) \int_{0}^{L_{z}} \frac{\partial^{2} T_{00}(u, v, w, \tau)}{\partial u^{2}}
$$$$
\times \frac{c_{n}(w) \mathrm{dwdv}}{T_{00}^{\varphi}(u, v, w, \tau)} \mathrm{d} u \mathrm{~d} \tau-T_{d}^{\varphi} \frac{2 \pi \alpha_{0 \text { ass }}}{L_{x} L_{y}^{2} L_{z}} \sum_{n=0}^{\infty} n C_{n}(x, y, z)
$$$$
\times e_{n T}(t) \int_{0}^{t} e_{n T}(-\tau) \int_{0}^{L_{x}} c_{n}(u) \int_{0}^{L_{y}} \int_{0}^{L_{z}} \frac{\partial^{2} T_{00}(u, v, w, \tau)}{\partial v^{2}}
$$$$
\times \frac{c_{n}(w) \mathrm{dws}_{\mathrm{n}}(\mathrm{v})}{T_{00}^{\varphi}(u, v, w, \tau)} \mathrm{d} v \mathrm{~d} u \mathrm{~d} \tau-T_{d}^{\varphi} \frac{2 \pi \alpha_{0 \text { ass }}}{L_{x} L_{y} L_{z}^{2}} \sum_{n=0}^{\infty} n C_{n}(x, y, z)
$$$$
\times \partial u \frac{\mathrm{dwdvdud} \tau}{T_{00}^{\varphi+1}(u, v, w, \tau)}-\varphi T_{d}^{\varphi} \frac{2 \pi \alpha_{0 \text { ass }}}{L_{x} L_{y}^{2} L_{z}} \sum_{n=0}^{\infty} C_{n}(x, y, z)
$$$$
\times e_{n T}(t) \int_{0}^{t} e_{n T}(-\tau) \int_{0}^{L_{x}} c_{n}(u) \int_{0}^{L_{y}} s_{n}(v) \int_{0}^{L_{z}} c_{n}(w)
$$$$
\times \frac{\partial T_{00}(u, v, w, \tau)}{\partial v} \frac{\partial T_{01}(u, v, w, \tau)}{\partial v} \frac{\text { dwdvdud } \tau}{T_{00}^{\varphi+1}(u, v, w, \tau)}
$$$$
-\varphi T_{d}^{\varphi} \frac{2 \pi \alpha_{0 \text { ass }}}{L_{x} L_{y} L_{z}^{2}} \sum_{n=0}^{\infty} C_{n}(x, y, z) e_{n T}(t) \int_{0}^{t} e_{n T}(-\tau)
$$$$
\times e_{n T}(t) \int_{0}^{t} e_{n T}(-\tau) \int_{0}^{L_{x}} c_{n}(u) \int_{0}^{L_{y}} c_{n}(v) \int_{0}^{L_{z}} s_{n}(w)
$$$$
\times \frac{\partial^{2} T_{00}(u, v, w, \tau)}{\partial w^{2}} \frac{\mathrm{dwdvdud} \tau}{T_{00}^{\varphi}(u, v, w, \tau)}-T_{d}^{\varphi} \frac{2 \varphi \alpha_{0 \text { ass }}}{L_{x} L_{y} L_{z}}
$$$$
\times \int_{0}^{L_{x}} c_{n}(u) \int_{0}^{L_{y}} c_{n}(v) \int_{0}^{L_{z}} \frac{\partial T_{00}(u, v, w, \tau)}{\partial v} \frac{\partial T_{01}(u, v, w, \tau)}{\partial v}
$$$$
\times \frac{s_{n}(w) \operatorname{dwdvdud} \tau}{T_{00}^{\varphi+1}(u, v, w, \tau)} ;
$$$$
\times \sum_{n=0}^{\infty} C_{n}(x, y, z) e_{n T}(t) \int_{0}^{t} e_{n T}(-\tau) \int_{0}^{L_{x}} c_{n}(u) \int_{0}^{L_{y}} c_{n}(v)
$$$$
\times \int_{0}^{L_{z}} c_{n}(w)\left\{\left[\frac{\partial T_{00}(u, v, w, \tau)}{\partial u}\right]^{2}+\left[\frac{\partial T_{00}(u, v, w, \tau)}{\partial v}\right]^{2}\right.
$$

$$
\begin{aligned}
& T_{11}(x, y, z, t)=\frac{2 \alpha_{0 \text { ass }}}{L_{x} L_{y} L_{z}} \sum_{n=0}^{\infty} C_{n}(x, y, z) e_{n T}(t) \int_{0}^{t} e_{n T}(-\tau) \\
& \quad \times \int_{0}^{L_{x}} c_{n}(u) \int_{0}^{L_{y}} c_{n}(v) \int_{0}^{L_{z}} c_{n}(w)\left[1+\frac{T_{01}(u, v, w, \tau)}{T_{00}(u, v, w, \tau)}\right] \\
& \times\left\{g_{T}(u) \frac{\partial^{2} T_{00}(u, v, w, \tau)}{\partial u^{2}}+g_{T}(u) \frac{\partial^{2} T_{00}(u, v, w, \tau)}{\partial v^{2}}\right.
\end{aligned}
$$

$\left.+\left[\frac{\partial T_{00}(u, v, w, \tau)}{\partial w}\right]^{2}\right\} \frac{\mathrm{dwdvdud} \tau}{T_{00}^{\varphi+1}(u, v, w, \tau)}$ 


$$
\begin{aligned}
& \left.+\frac{\partial}{\partial w}\left[g_{T}(w) \frac{\partial T_{00}(u, v, w, \tau)}{\partial w}\right]\right\} \mathrm{d} w \mathrm{~d} v \mathrm{~d} u \mathrm{~d} \tau \\
& +T_{d}^{\varphi} \frac{2 \alpha_{0 \text { ass }}}{L_{x} L_{y} L_{z}} \sum_{n=0}^{\infty} C_{n}(x, y, z) e_{n T}(t) \int_{0}^{t} e_{n T}(-\tau) \\
& \times \int_{0}^{L_{x}} c_{n}(u) \int_{0}^{L_{y}} \int_{0}^{L_{z}}\left[\frac{\partial^{2} T_{10}(u, v, w, \tau)}{\partial u^{2}}+\frac{\partial^{2} T_{10}(u, v, w, \tau)}{\partial v^{2}}\right. \\
& \left.+\frac{\partial^{2} T_{10}(u, v, w, \tau)}{\partial w^{2}}\right] c_{n}(w) c_{n}(v) \frac{\mathrm{dwdvdud} \tau}{T_{00}^{\varphi}(u, v, w, \tau)} \\
& +T_{d}^{\varphi} \frac{2 \alpha_{0 \mathrm{ass}}}{L_{x} L_{y} L_{z}} \sum_{n=0}^{\infty} C_{n}(x, y, z) e_{n T}(t) \int_{0}^{t} e_{n T}(-\tau) \\
& \times \int_{0}^{L_{x}} c_{n}(u) \int_{0}^{L_{y}} c_{n}(v) \int_{0}^{L_{z}} c_{n}(w)\left\{g_{T}(w) \frac{\partial T_{10}(u, v, w, \tau)}{\partial u}\right. \\
& \times \frac{\partial T_{00}(u, v, w, \tau)}{\partial u}+g_{T}(w) \frac{\partial T_{10}(u, v, w, \tau)}{\partial v} \\
& \times \frac{\partial T_{00}(u, v, w, \tau)}{\partial v}+\frac{\partial T_{10}(u, v, w, \tau)}{\partial w} \\
& \left.\times \frac{\partial}{\partial w}\left[g_{T}(w) \frac{\partial T_{00}(u, v, w, \tau)}{\partial w}\right]\right\} \frac{\mathrm{dwdvdud} \tau}{T_{00}^{\varphi+1}(u, v, w, \tau)} \\
& -T_{d}^{\varphi} \frac{2 \alpha_{0 \mathrm{ass}}}{L_{x} L_{y} L_{z}} \sum_{n=0}^{\infty} C_{n}(x, y, z) e_{n T}(t) \int_{0}^{t} e_{n T}(-\tau) \int_{0}^{L_{x}} c_{n}(u) \\
& \times \int_{0}^{L_{y}} c_{n}(v) \int_{0}^{L_{z}} c_{n}(w)\left[\frac{\partial T_{10}(u, v, w, \tau)}{\partial u} \frac{\partial T_{00}(u, v, w, \tau)}{\partial u}\right. \\
& +\frac{\partial T_{10}(u, v, w, \tau)}{\partial v} \frac{\partial T_{00}(u, v, w, \tau)}{\partial v}+\frac{\partial T_{10}(u, v, w, \tau)}{\partial w} \\
& \left.\times \frac{\partial T_{00}(u, v, w, \tau)}{\partial w}\right] \frac{\mathrm{dwdvdud} \tau}{T_{00}^{\varphi+1}(u, v, w, \tau)}-T_{d}^{\varphi} \frac{2 \alpha_{0 \text { ass }}}{L_{x} L_{y} L_{z}} \\
& \times \alpha_{0 \text { ass }} \sum_{n=0}^{\infty} C_{n}(x, y, z) e_{n T}(t) \int_{0}^{t} e_{n T}(-\tau) \int_{0}^{L_{x}} g_{T}(u) c_{n}(u) \\
& \times \int_{0}^{L_{y}} c_{n}(v) \int_{0}^{L_{w}} c_{n}(w)\left\{\left[\frac{\partial T_{00}(u, v, w, \tau)}{\partial u}\right]^{2}\right. \\
& \left.+\left[\frac{\partial T_{00}(u, v, w, \tau)}{\partial v}\right]^{2}+\left[\frac{\partial T_{00}(u, v, w, \tau)}{\partial w}\right]^{2}\right\} \frac{T_{d}^{\varphi} \operatorname{dwdvdud} \tau}{T_{00}^{\varphi+1}(u, v, w, \tau)} . \\
& -\frac{t}{6} \int_{0}^{\infty} \int_{0}^{z}\left[\frac{\partial^{2} u_{x 1}(x, y, w, \tau)}{\partial x^{2}}-\frac{\partial^{2} u_{y 1}(x, y, w, \tau)}{\partial x \partial y}\right. \\
& \left.-\frac{\partial^{2} u_{z 1}(x, y, w, \tau)}{\partial x \partial w}\right] \frac{E(w) \mathrm{dw}}{1+\sigma(w)} \mathrm{d} \tau \\
& -\frac{t}{2} \int_{0}^{\infty} \int_{0}^{z}\left[\frac{\partial^{2} u_{y 1}(x, y, w, \tau)}{\partial y^{2}}-\frac{\partial^{2} u_{x 1}(x, y, w, \tau)}{\partial x \partial y}\right] \\
& \times \frac{E(w) \mathrm{dw}}{1+\sigma(w)} \mathrm{d} \tau+\frac{1}{2} \int_{0}^{t} \int_{0}^{z}\left[\frac{\partial^{2} u_{y 1}(x, y, w, \tau)}{\partial y^{2}}\right. \\
& \left.-\frac{\partial^{2} u_{x 1}(x, y, w, \tau)}{\partial x \partial y}\right] \frac{E(w) \mathrm{dw}}{1+\sigma(w)}(t-\tau) \mathrm{d} \tau \\
& -\frac{t}{2} \int_{0}^{\infty} \int_{0}^{z}\left[\frac{\partial^{2} u_{y 1}(x, y, w, \tau)}{\partial w^{2}}-\frac{\partial^{2} u_{x 1}(x, y, w, \tau)}{\partial x \partial w}\right] \\
& \times \frac{E(w) \mathrm{d} w}{1+\sigma(w)} \mathrm{d} \tau+\frac{1}{2} \int_{0}^{t}(t-\tau) \int_{0}^{z}\left[\frac{\partial^{2} u_{y 1}(x, y, w, \tau)}{\partial w^{2}}\right. \\
& \left.-\frac{\partial^{2} u_{x 1}(x, y, w, \tau)}{\partial x \partial w}\right] \frac{E(w) \mathrm{d} w}{1+\sigma(w)} \mathrm{d} \tau+\int_{0}^{t} \int_{0}^{z}\left[\frac{\partial^{2} u_{x 1}(x, y, w, \tau)}{\partial x^{2}}\right. \\
& \left.+\frac{\partial^{2} u_{y 1}(x, y, w, \tau)}{\partial x \partial y}+\frac{\partial^{2} u_{z 1}(x, y, w, \tau)}{\partial x \partial w}\right] K(w) \mathrm{d} w(t-\tau) \mathrm{d} \tau \\
& -t \int_{0}^{\infty} \int_{0}^{z}\left[\frac{\partial^{2} u_{x 1}(x, y, w, \tau)}{\partial x^{2}}+\frac{\partial^{2} u_{y 1}(x, y, w, \tau)}{\partial x \partial y}\right. \\
& \left.+\frac{\partial^{2} u_{z 1}(x, y, w, \tau)}{\partial x \partial w}\right] K(w) \mathrm{d} w \mathrm{~d} \tau-\int_{0}^{(t-\tau)} \int_{0}^{z} K(w) \chi(w) \\
& \times \frac{\partial T(x, y, w, \tau)}{\partial x} \mathrm{~d} w \mathrm{~d} \tau+t \int_{0}^{\infty} \int_{0}^{z} K(w) \chi(w) \\
& \times \frac{\partial T(x, y, w, \tau)}{\partial x} \mathrm{~d} w \mathrm{~d} \tau-\int_{0}^{t}(t-\tau) \int_{0}^{z} \rho(w) \\
& \times \frac{\partial^{2} u_{x 1}(x, y, w, \tau)}{\partial \tau^{2}} \mathrm{~d} w \mathrm{~d} \tau+t \int_{0}^{\infty} \int_{0}^{z} \rho(w) \frac{\partial^{2} u_{x 1}(x, y, w, \tau)}{\partial \tau^{2}} \\
& \left.\times \mathrm{d} w \mathrm{~d} \tau-\Phi_{x 1}(x, y, z, t)-\alpha_{u x 2} \Phi_{x 0}(x, y, z, t)\right\} ;
\end{aligned}
$$

The second-order approximations of components of the displacement vector

$$
\begin{aligned}
& u_{x 2}(x, y, z, t)=\alpha_{u x 2}+u_{x 1}(x, y, z, t) \\
& +\phi_{1}\left\{\frac { 1 } { 6 } \int _ { 0 } ^ { t } \int _ { 0 } ^ { z } \left[\frac{\partial^{2} u_{x 1}(x, y, w, \tau)}{\partial x^{2}}-\frac{\partial^{2} u_{y 1}(x, y, w, \tau)}{\partial x \partial y}\right.\right. \\
& \left.-\frac{\partial^{2} u_{z 1}(x, y, w, \tau)}{\partial x \partial w}\right] \frac{E(w) \mathrm{dw}}{1+\sigma(w)}(t-\tau) \mathrm{d} \tau
\end{aligned}
$$

$$
\begin{aligned}
& u_{y 2}(x, y, z, t)=\alpha_{u y 2}+u_{y 1}(x, y, z, t)+\phi_{1}\left\{\frac{1}{2} \int_{0}^{t}(t-\tau)\right. \\
& \times \int_{0}^{z}\left[\frac{\partial^{2} u_{y 1}(x, y, w, \tau)}{\partial y^{2}}+\frac{\partial^{2} u_{x 1}(x, y, w, \tau)}{\partial x \partial y}\right] \frac{E(w) \mathrm{dw}}{1+\sigma(w)} \mathrm{d} \tau \\
& -\frac{t}{2} \int_{0}^{t} \int_{0}^{z}\left[\frac{\partial^{2} u_{y 1}(x, y, w, \tau)}{\partial y^{2}}+\frac{\partial^{2} u_{x 1}(x, y, w, \tau)}{\partial x \partial y}\right] \frac{E(w) \mathrm{dw}}{1+\sigma(w)} \mathrm{d} \tau
\end{aligned}
$$


Appl Nanosci (2012) 2:71-89

81

$$
\begin{aligned}
& +\frac{1}{6} \int_{0}^{t}(t-\tau) \int_{0}^{z}\left[5 \frac{\partial^{2} u_{y 1}(x, y, w, \tau)}{\partial y^{2}}-\frac{\partial^{2} u_{x 1}(x, y, w, \tau)}{\partial x \partial y}\right. \\
& \left.+\frac{\partial^{2} u_{y 1}(x, y, w, \tau)}{\partial y \partial w}\right] \frac{E(w) \mathrm{dw}}{1+\sigma(w)} \mathrm{d} \tau-\int_{0}^{\infty} \int_{0}^{z}\left[\frac{\partial^{2} u_{z 1}(x, y, w, \tau)}{\partial y^{2}}\right. \\
& \left.-\frac{\partial^{2} u_{z 1}(x, y, w, \tau)}{\partial y \partial w}\right] \frac{E(w) \mathrm{d} w}{1+\sigma(w)} \mathrm{d} \tau-\frac{t}{6} \int_{0}^{\infty} \int_{0}^{z} \frac{E(w)}{1+\sigma(w)} \\
& \left.+\frac{\partial^{2} u_{y 1}(x, y, w, \tau)}{\partial y \partial w}\right] \frac{t}{2} \frac{E(w) \mathrm{dw}}{1+\sigma(w)} \mathrm{d} \tau+\frac{E(z)}{6[1+\sigma(w)]} \int_{0}^{t}(t-\tau) \\
& \times\left[5 \frac{\partial^{2} u_{y 1}(x, y, w, \tau)}{\partial y^{2}}-\frac{\partial^{2} u_{x 1}(x, y, w, \tau)}{\partial x \partial y}-\frac{\partial^{2} u_{z 1}(x, y, w, \tau)}{\partial y \partial w}\right] \\
& \times\left[5 \frac{\partial u_{z 1}(x, y, z, \tau)}{\partial z}-\frac{\partial u_{x 1}(x, y, z, \tau)}{\partial x}-\frac{\partial u_{y 1}(x, y, z, \tau)}{\partial y}\right] \mathrm{d} \tau \\
& \times \mathrm{d} w \mathrm{~d} \tau+\frac{E(z)}{2[1+\sigma(z)]} \int_{0}^{t}(t-\tau)\left[\frac{\partial u_{y 1}(x, y, z, \tau)}{\partial z}\right. \\
& -\frac{t E(z)}{6[1+\sigma(w)]} \int_{0}^{\infty}\left[5 \frac{\partial u_{z 1}(x, y, z, \tau)}{\partial z}-\frac{\partial u_{x 1}(x, y, z, \tau)}{\partial x}\right. \\
& \left.+\frac{\partial u_{z 1}(x, y, z, \tau)}{\partial y}\right] \mathrm{d} \tau-\frac{t E(z)}{2[1+\sigma(z)]} \int_{0}^{\infty}\left[\frac{\partial u_{y 1}(x, y, z, \tau)}{\partial z}\right. \\
& \left.-\frac{\partial u_{y 1}(x, y, z, \tau)}{\partial y}\right] \mathrm{d} \tau+\int_{0}^{t}\left[\frac{\partial u_{x 1}(x, y, z, \tau)}{\partial z}+\frac{\partial u_{y 1}(x, y, z, \tau)}{\partial y}\right. \\
& \left.+\frac{\partial u_{z 1}(x, y, z, \tau)}{\partial y}\right] \mathrm{d} \tau+\int_{0}^{t}(t-\tau) \int_{0}^{z} K(w)\left[\frac{\partial^{2} u_{y 1}(x, y, w, \tau)}{\partial y^{2}}\right. \\
& \left.+\frac{\partial u_{z 1}(x, y, z, \tau)}{\partial z}\right](t-\tau) \mathrm{d} \tau K(z)-t K(z) \int_{0}^{\infty}\left[\frac{\partial u_{x 1}(x, y, z, \tau)}{\partial z}\right. \\
& \left.+\frac{\partial^{2} u_{y 1}(x, y, w, \tau)}{\partial x \partial y}+\frac{\partial^{2} u_{y 1}(x, y, w, \tau)}{\partial y \partial w}\right] \mathrm{d} w \mathrm{~d} \tau \\
& \left.+\frac{\partial u_{y 1}(x, y, z, \tau)}{\partial y}+\frac{\partial u_{z 1}(x, y, z, \tau)}{\partial z}\right] \mathrm{d} \tau-\int_{0}^{t}(t-\tau) \\
& -t \int_{0}^{\infty} \int_{0}^{z} K(w)\left[\frac{\partial^{2} u_{y 1}(x, y, w, \tau)}{\partial y^{2}}+\frac{\partial^{2} u_{y 1}(x, y, w, \tau)}{\partial x \partial y}\right. \\
& \times \int_{0}^{z} K(w) \chi(w) \frac{\partial T(x, y, w, \tau)}{\partial w} \mathrm{~d} w \mathrm{~d} \tau+t \int_{0}^{\infty} \int_{0}^{z} K(w) \\
& \left.+\frac{\partial^{2} u_{y 1}(x, y, w, \tau)}{\partial y \partial w}\right] \mathrm{d} w \mathrm{~d} \tau-\int_{0}^{t}(t-\tau) \int_{0}^{z} K(w) \chi(w) \\
& \left.\times \frac{\partial T(x, y, w, \tau)}{\partial w} \chi(w) \mathrm{d} w \mathrm{~d} \tau-\alpha_{u z 2} \Phi_{z 0}(x, y, z, t)-\Phi_{z 1}(x, y, z, t)\right\} . \\
& \times \frac{\partial T(x, y, w, \tau)}{\partial y} \mathrm{~d} w \mathrm{~d} \tau+t \int_{0}^{\infty} \int_{0}^{z} K(w) \chi(w) \frac{\partial T(x, y, w, \tau)}{\partial y}
\end{aligned}
$$$$
\times \mathrm{d} w \mathrm{~d} \tau-\int_{0}^{t}(t-\tau) \int_{0}^{z} \rho(w) \frac{\partial^{2} u_{y 1}(x, y, w, \tau)}{\partial \tau^{2}} \mathrm{~d} w \mathrm{~d} \tau
$$$$
+t \int_{0}^{\infty} \int_{0}^{z} \rho(w) \frac{\partial^{2} u_{y 1}(x, y, w, \tau)}{\partial \tau^{2}} \mathrm{~d} w \mathrm{~d} \tau-\alpha_{u y 2} \Phi_{y 0}(x, y, z, t)
$$$$
\left.-\Phi_{y 1}(x, y, z, t)\right\}
$$

Calculation of the parameters $\alpha_{u \beta 2}$ leads to the following results

$$
\begin{aligned}
& \alpha_{u x 2}=L_{z} \frac{1}{12} \int_{0}^{\Theta}(\Theta-t)^{2} \int_{-L_{x}}^{L_{x}} \int_{-L_{y}}^{L_{y}} \int_{0}^{L_{z}}\left(L_{z}-z\right) \\
& \times {\left[5 \frac{\partial^{2} u_{x 1}(x, y, z, t)}{\partial x^{2}}-\frac{\partial^{2} u_{y 1}(x, y, z, t)}{\partial x \partial y}-\frac{\partial^{2} u_{y 1}(x, y, z, t)}{\partial x \partial z}\right] } \\
& \times \frac{E(z) \mathrm{d} z}{1+\sigma(z)} \mathrm{d} y \mathrm{~d} x d t-\frac{\Theta^{2}}{12} \int_{0}^{\infty} \int_{-L_{x}}^{L_{x}} \int_{-L_{y}}^{L_{y}} \int_{0}^{L_{z}}\left(L_{z}-z\right) \\
& \times\left[5 \frac{\partial^{2} u_{x 1}(x, y, z, t)}{\partial x^{2}}-\frac{\partial^{2} u_{y 1}(x, y, z, t)}{\partial x \partial y}-\frac{\partial^{2} u_{y 1}(x, y, z, t)}{\partial x \partial z}\right] \\
& \times \frac{E(z) \mathrm{dz}}{1+\sigma(z)} \mathrm{d} y \mathrm{~d} x d t+\frac{1}{2} \int_{0}^{\Theta} \int_{-L_{x}}^{L_{x}} \int_{-L_{y}}^{L_{y}} \int_{0}^{L_{z}}\left(L_{z}-z\right) \\
& \times\left[\frac{\partial^{2} u_{y 1}(x, y, z, t)}{\partial y^{2}}+\frac{\partial^{2} u_{x 1}(x, y, z, t)}{\partial x \partial y} \frac{E(z) \mathrm{d} z}{1+\sigma(z)} \mathrm{d} y \mathrm{~d} x\right. \\
& \times(\Theta-t)^{2} \mathrm{~d} t-\frac{\Theta^{2}}{2} \int_{0}^{\infty} \int_{-L_{x}}^{L_{x}} \int_{-L_{y}}^{L_{y}} \int_{0}^{L_{z}}\left(L_{z}-z\right)
\end{aligned}
$$

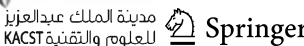




$$
\begin{aligned}
& \times\left[\frac{\partial^{2} u_{y 1}(x, y, z, t)}{\partial y^{2}}+\frac{\partial^{2} u_{x 1}(x, y, z, t)}{\partial x \partial y}\right] \frac{E(z) \mathrm{d} z}{1+\sigma(z)} \mathrm{d} y \mathrm{~d} x \mathrm{~d} t \\
& -\frac{\Theta^{2}}{2} \int_{0}^{\infty} \int_{-L_{x}}^{L_{x}} \int_{-L_{y}}^{L_{y}} \int_{0}^{L_{z}}\left(L_{z}-z\right)\left[\frac{\partial^{2} u_{y 1}(x, y, z, t)}{\partial z^{2}}\right. \\
& \left.+\frac{\partial^{2} u_{x 1}(x, y, z, t)}{\partial x \partial z}\right] \frac{E(z) \mathrm{d} z}{1+\sigma(z)} \mathrm{d} y \mathrm{~d} x \mathrm{~d} t+\frac{1}{2} \int_{0}^{\Theta}(\Theta-t)^{2} \\
& \times \int_{-L_{x}}^{L_{x}} \int_{-L_{y}}^{L_{y}} \int_{0}^{L_{z}}\left(L_{z}-z\right)\left[\frac{\partial^{2} u_{y 1}(x, y, z, t)}{\partial z^{2}}+\frac{\partial^{2} u_{x 1}(x, y, z, t)}{\partial x \partial z}\right] \\
& \times \frac{E(z) \mathrm{d} z}{1+\sigma(z)} \mathrm{d} y \mathrm{~d} x \mathrm{~d} t-\frac{\Theta^{2}}{2} \int_{0}^{\infty} \int_{-L_{x}}^{L_{x}} \int_{-L_{y}}^{L_{y}} \int_{0}^{L_{z}} K(z) \\
& \times \int_{-L_{x}}^{L_{x}} \int_{-L_{y}}^{L_{y}} \int_{0}^{L_{z}}\left(L_{z}-z\right)\left[\frac{\partial^{2} u_{y 1}(x, y, z, t)}{\partial z^{2}}+\frac{\partial^{2} u_{x 1}(x, y, z, t)}{\partial x \partial z}\right] \\
& \times \frac{E(z) \mathrm{dz}}{1+\sigma(z)} \mathrm{d} y \mathrm{~d} x \mathrm{~d} t-\frac{\Theta^{2}}{2} \int_{0}^{\infty} \int_{-L_{x}}^{L_{x}} \int_{-L_{y}}^{L_{y}} \int_{0}^{L_{z}} K(z) \\
& \times\left(L_{z}-z\right)\left[\frac{\partial^{2} u_{x 1}(x, y, z, t)}{\partial x^{2}}+\frac{\partial^{2} u_{y 1}(x, y, z, t)}{\partial x \partial y}\right. \\
& \left.+\frac{\partial^{2} u_{z 1}(x, y, z, t)}{\partial x \partial z}\right] \mathrm{d} z \mathrm{~d} y \mathrm{~d} x \mathrm{~d} t+\frac{1}{2} \int_{0}^{\Theta}(\Theta-t)^{2} \\
& \times \int_{-L_{x}}^{L_{x}} \int_{-L_{y}}^{L_{y}} \int_{0}^{L_{z}}\left(L_{z}-z\right) K(z)\left[\frac{\partial^{2} u_{x 1}(x, y, z, t)}{\partial x^{2}}\right. \\
& \left.+\frac{\partial^{2} u_{y 1}(x, y, z, t)}{\partial x \partial y}+\frac{\partial^{2} u_{z 1}(x, y, z, t)}{\partial x \partial z}\right] \mathrm{d} z \mathrm{~d} y \mathrm{~d} x \mathrm{~d} t \\
& -\frac{1}{2} \int_{0}^{\Theta}(\Theta-t)^{2} \int_{-L_{x}}^{L_{x}} \int_{-L_{y}}^{L_{y}} \int_{0}^{L_{z}}\left(L_{z}-z\right) \rho(z) \frac{\partial^{2} u_{x 1}(x, y, z, t)}{\partial \tau^{2}} \\
& \times \mathrm{d} z \mathrm{~d} y \mathrm{~d} x \mathrm{~d} t+\frac{\Theta^{2}}{2} \int_{0}^{\Theta} \int_{-L_{x}}^{L_{x}} \int_{-L_{y}}^{L_{y}} \int_{0}^{L_{z}}\left(L_{z}-z\right) \\
& \times \rho(z) \frac{\partial^{2} u_{x 1}(x, y, z, t)}{\partial \tau^{2}} \mathrm{~d} z \mathrm{~d} y \mathrm{~d} x \mathrm{~d} t-\int_{0}^{\Theta} \int_{-L_{x}}^{L_{x}} \int_{-L_{y}}^{L_{y}} \int_{0}^{L_{z}}\left(L_{z}-z\right) \\
& \times \rho(z) u_{x 1}(x, y, z, t) \mathrm{d} z \mathrm{~d} y \mathrm{~d} x \mathrm{~d} t \\
& \left.-\frac{\Xi_{x 2}(\Theta)}{2}+\Theta^{2} \frac{\Xi_{x 0}(\infty)}{2}\right\} / 4 L \Theta \Lambda \\
& \begin{array}{l}
\alpha_{u y 2}=L_{z}\left\{\frac { 1 } { 2 } \int _ { 0 } ^ { \Theta } \int _ { - L _ { x } } ^ { L _ { x } } \int _ { - L _ { y } } ^ { L _ { y } } \int _ { 0 } ^ { L _ { z } } ( L _ { z } - z ) \left[\frac{\partial^{2} u_{y 1}(x, y, z, t)}{\partial x^{2}}\right.\right. \\
\left.+\frac{\partial^{2} u_{x 1}(x, y, z, t)}{\partial x \partial y}\right] \frac{E(z) \mathrm{d} z}{1+\sigma(z)} \mathrm{d} y \mathrm{~d} x .
\end{array} \\
& \times(\Theta-t)^{2} \mathrm{~d} t-\frac{\Theta^{2}}{2} \int_{0}^{\infty} \int_{-L_{x}}^{L_{x}} \int_{-L_{y}}^{L_{y}} \int_{0}^{L_{z}}\left(L_{z}-z\right) \\
& \times\left[\frac{\partial^{2} u_{y 1}(x, y, z, t)}{\partial x^{2}}+\frac{\partial^{2} u_{x 1}(x, y, z, t)}{\partial x \partial y}\right] \frac{E(z) \mathrm{d} z}{1+\sigma(z)} \\
& \times E(z) \mathrm{d} z \mathrm{~d} y \mathrm{~d} x \mathrm{~d} t+\frac{1}{12} \int_{0}^{\Theta}(\Theta-t)^{2} \int_{-L_{x}}^{L_{x}} \int_{-L_{y}}^{L_{y}} \int_{0}^{L_{z}}\left(L_{z}-z\right) \\
& \times\left[5 \frac{\partial^{2} u_{y 1}(x, y, z, t)}{\partial y^{2}}-\frac{\partial^{2} u_{x 1}(x, y, z, t)}{\partial x \partial y}-\frac{\partial^{2} u_{z 1}(x, y, z, t)}{\partial y \partial z}\right] \\
& \times \frac{E(z) \mathrm{d} z}{1+\sigma(z)} \mathrm{d} y \mathrm{~d} x \mathrm{~d} t-\frac{\Theta^{2}}{12} \int_{0}^{\infty} \int_{-L_{x}}^{L_{x}} \int_{-L_{y}}^{L_{y}} \int_{0}^{L_{z}}\left[5 \frac{\partial^{2} u_{y 1}(x, y, z, t)}{\partial y^{2}}\right. \\
& \left.-\frac{\partial^{2} u_{x 1}(x, y, z, t)}{\partial x \partial y}-\frac{\partial^{2} u_{z 1}(x, y, z, t)}{\partial y \partial z}\right]\left(L_{z}-z\right) \\
& \times \frac{E(z) \mathrm{d} z}{1+\sigma(z)} \mathrm{d} y \mathrm{~d} x \mathrm{~d} t+\frac{1}{2} \int_{0}^{\Theta} \int_{-L_{x}}^{L_{x}} \int_{-L_{y}}^{L_{y}} \int_{0}^{L_{z}}\left(L_{z}-z\right) \\
& \times\left[\frac{\partial u_{y 1}(x, y, z, t)}{\partial z}+\frac{\partial u_{z 1}(x, y, z, t)}{\partial y}\right] \frac{E(z) \mathrm{d} z}{1+\sigma(z)} \mathrm{d} y \mathrm{~d} x \\
& \times(\Theta-t)^{2} \mathrm{~d} t-\frac{\Theta^{2}}{2} \int_{0}^{\infty} \int_{-L_{x}}^{L_{x}} \int_{-L_{y}}^{L_{y}} \int_{0}^{L_{z}}\left(L_{z}-z\right)\left[\frac{\partial u_{y 1}(x, y, z, t)}{\partial z}\right. \\
& \left.+\frac{\partial u_{z 1}(x, y, z, t)}{\partial y}\right] \frac{E(z) \mathrm{dz}}{1+\sigma(z)} \mathrm{d} y \mathrm{~d} x \mathrm{~d} t+\frac{1}{2} \int_{0}^{\Theta}(\Theta-t)^{2} \\
& \times \int_{-L_{x}}^{L_{x}} \int_{-L_{y}}^{L_{y}} \int_{0}^{L_{z}}\left(L_{z}-z\right) K(z)\left[\frac{\partial u_{y 1}(x, y, z, t)}{\partial y^{2}}+\frac{\partial u_{y 1}(x, y, z, t)}{\partial x \partial y}\right. \\
& \left.+\frac{\partial u_{y 1}(x, y, z, t)}{\partial y \partial z}\right] \mathrm{d} z \mathrm{~d} y \mathrm{~d} x \mathrm{~d} t-\frac{\Theta^{2}}{2} \int_{0}^{\infty} \int_{-L_{x}}^{L_{x}} \int_{-L_{y}}^{L_{y}} \int_{0}^{L_{z}}\left(L_{z}-z\right) \\
& \times\left[\frac{\partial u_{y 1}(x, y, z, t)}{\partial y^{2}}+\frac{\partial u_{y 1}(x, y, z, t)}{\partial x \partial y}+\frac{\partial u_{y 1}(x, y, z, t)}{\partial y \partial z}\right] \\
& \times K(z) \mathrm{d} z \mathrm{~d} y \mathrm{~d} x \mathrm{~d} t-\frac{1}{2} \int_{0}^{\Theta}(\Theta-t)^{2} \int_{-L_{x}}^{L_{x}} \int_{-L_{y}}^{L_{y}} \int_{0}^{L_{z}} \frac{\partial u_{y 1}(x, y, z, t)}{\partial t^{2}}
\end{aligned}
$$




$$
\begin{aligned}
& \times \rho(z) \mathrm{d} z \mathrm{~d} y \mathrm{~d} x \mathrm{~d} t+\frac{\Theta^{2}}{2} \int_{0}^{\Theta} \int_{-L_{x}}^{L_{x}} \int_{-L_{y}}^{L_{y}} \int_{0}^{L_{z}} \rho(z) \frac{\partial u_{y 1}(x, y, z, t)}{\partial t^{2}} \quad \times \frac{E(z) \mathrm{d} z}{1+\sigma(z)} \mathrm{d} y \mathrm{~d} x \mathrm{~d} t-\frac{\Theta^{2}}{2} \int_{0}^{\infty} \int_{-L_{x}}^{L_{x}} \int_{-L_{y}}^{L_{y}} \int_{0}^{L_{z}} K(z) \\
& \times \mathrm{d} z \mathrm{~d} y \mathrm{~d} x \mathrm{~d} t-\int_{0}^{\Theta} \int_{-L_{x}}^{L_{x}} \int_{-L_{y}}^{L_{y}} \int_{0}^{L_{z}}\left(L_{z}-z\right) \rho(z) \\
& \left.\times u_{y 1}(x, y, z, t) \mathrm{d} z \mathrm{~d} y \mathrm{~d} x \mathrm{~d} t-\frac{\Xi_{y 2}(\Theta)}{2}+\Theta^{2} \frac{\Xi_{y 0}(\infty)}{2}\right\} / 4 L \Theta \Lambda \\
& \times\left[\frac{\partial u_{x 1}(x, y, z, t)}{\partial x}+\frac{\partial u_{y 1}(x, y, z, t)}{\partial y}+\frac{\partial u_{z 1}(x, y, z, t)}{\partial z}\right] \\
& \times \frac{E(z) \mathrm{d} z}{1+\sigma(z)} \mathrm{d} y \mathrm{~d} x \mathrm{~d} t-\int_{0}^{\Theta} \int_{-L_{x}}^{L_{x}} \int_{-L_{y}}^{L_{y}} \int_{0}^{L_{z}}\left(L_{z}-z\right) \\
& \left.\times \rho(z) u_{z 1}(x, y, z, t) \mathrm{d} z \mathrm{~d} y \mathrm{~d} x \mathrm{~d} t-\frac{\Xi_{z 2}(\Theta)}{2}+\Theta^{2} \frac{\Xi_{z 0}(\infty)}{2}\right\} / 4 L \Theta \Lambda . \\
& \alpha_{u z 2}=L_{z}\left\{\frac{1}{2} \int_{0}^{\Theta}(\Theta-t)^{2} \int_{-L_{x}}^{L_{x}} \int_{-L_{y}}^{L_{y}} \int_{0}^{L_{z}}\left(L_{z}-z\right)\right. \\
& \times\left[\frac{\partial^{2} u_{z 1}(x, y, z, t)}{\partial x^{2}}+\frac{\partial^{2} u_{x 1}(x, y, z, t)}{\partial x \partial z}\right] \frac{E(z) \mathrm{d} z}{1+\sigma(z)} \mathrm{d} y \mathrm{~d} x \mathrm{~d} t \\
& -\frac{\Theta^{2}}{2} \int_{0}^{\infty} \int_{-L_{x}}^{L_{x}} \int_{-L_{y}}^{L_{y}} \int_{0}^{L_{z}}\left(L_{z}-z\right)\left[\frac{\partial^{2} u_{z 1}(x, y, z, t)}{\partial x^{2}}\right. \\
& \left.+\frac{\partial^{2} u_{x 1}(x, y, z, t)}{\partial x \partial z}\right] \frac{E(z) \mathrm{d} z}{1+\sigma(z)} \mathrm{d} y \mathrm{~d} x \mathrm{~d} t+\frac{1}{2} \int_{0}^{\Theta}(\Theta-t)^{2} \\
& \times \int_{-L_{x}}^{L_{x}} \int_{-L_{y}}^{L_{y}} \int_{0}^{L_{z}}\left(L_{z}-z\right)\left[\frac{\partial^{2} u_{z 1}(x, y, z, t)}{\partial y^{2}}+\frac{\partial^{2} u_{y 1}(x, y, z, t)}{\partial y \partial z}\right] \\
& \times \frac{E(z) \mathrm{dz}}{1+\sigma(z)} \mathrm{d} y \mathrm{~d} x \mathrm{~d} t-\int_{0}^{\Theta} \int_{-L_{x}}^{L_{x}} \int_{-L_{y}}^{L_{y}} \int_{0}^{L_{z}}\left(L_{z}-z\right) \\
& \times \frac{\Theta^{2}}{2}\left[\frac{\partial^{2} u_{z 1}(x, y, z, t)}{\partial y^{2}}+\frac{\partial^{2} u_{y 1}(x, y, z, t)}{\partial y \partial z}\right] \frac{E(z) \mathrm{d} z}{1+\sigma(z)} \mathrm{d} y \mathrm{~d} x \mathrm{~d} t \\
& +\frac{1}{12} \int_{0}^{\Theta}(\Theta-t)^{2} \int_{-L_{x}}^{L_{x}} \int_{-L_{y}}^{L_{y}} \int_{0}^{L_{z}}\left(L_{z}-z\right) \\
& \times\left[5 \frac{\partial u_{z 1}(x, y, z, t)}{\partial z}-\frac{\partial u_{x 1}(x, y, z, t)}{\partial x}-\frac{\partial u_{y 1}(x, y, z, t)}{\partial y}\right] \\
& \times \frac{E(z) \mathrm{dz}}{1+\sigma(z)} \mathrm{d} y \mathrm{~d} x \mathrm{~d} t-\frac{\Theta^{2}}{12} \int_{0}^{\Theta} \int_{-L_{x}}^{L_{x}} \int_{-L_{y}}^{L_{y}} \int_{0}^{L_{z}}\left(L_{z}-z\right) \\
& \times\left[5 \frac{\partial u_{z 1}(x, y, z, t)}{\partial z}-\frac{\partial u_{x 1}(x, y, z, t)}{\partial x}-\frac{\partial u_{y 1}(x, y, z, t)}{\partial y}\right] \\
& \times \frac{E(z) \mathrm{dz}}{1+\sigma(z)} \mathrm{d} y \mathrm{~d} x \mathrm{~d} t+\frac{1}{2} \int_{0}^{\Theta}(\Theta-t)^{2} \int_{-L_{x}}^{L_{x}} \int_{-L_{y}}^{L_{y}} \int_{0}^{L_{z}} K(z) \\
& \times\left[\frac{\partial u_{x 1}(x, y, z, t)}{\partial x}+\frac{\partial u_{y 1}(x, y, z, t)}{\partial y}+\frac{\partial u_{z 1}(x, y, z, t)}{\partial z}\right] \\
& \frac{\partial C_{i 00}(x, y, z, t)}{\partial t}=D_{0 L} \frac{\partial^{2} C_{i 00}(x, y, z, t)}{\partial x^{2}}+D_{0 L} \frac{\partial^{2} C_{i 00}(x, y, z, t)}{\partial y^{2}} \\
& +D_{0 L} \frac{\partial^{2} C_{i 00}(x, y, z, t)}{\partial z^{2}}+D_{0 L} \frac{\partial}{\partial x}\left[g_{L}(z, T) \frac{\partial C_{i-100}(x, y, z, t)}{\partial x}\right] \\
& +D_{0 L} \frac{\partial}{\partial y}\left[g_{L}(z, T) \frac{\partial C_{i-100}(x, y, z, t)}{\partial y}\right] \\
& +D_{0 L} \frac{\partial}{\partial z}\left[g_{L}(z, T) \frac{\partial C_{i-100}(x, y, z, t)}{\partial z}\right], i \geq 1 ; \\
& \frac{\partial C_{010}(x, y, z, t)}{\partial t}=D_{0 L} \frac{\partial^{2} C_{010}(x, y, z, t)}{\partial x^{2}}+D_{0 L} \frac{\partial^{2} C_{010}(x, y, z, t)}{\partial y^{2}} \\
& +D_{0 L} \frac{\partial^{2} C_{010}(x, y, z, t)}{\partial z^{2}}+D_{0 L} \frac{\partial}{\partial x}\left[\frac{C_{000}^{\gamma}(x, y, z, t)}{P^{\gamma}(z, T)}\right. \\
& \left.\times \frac{\partial C_{000}(x, y, z, t)}{\partial x}\right]+D_{0 L} \frac{\partial}{\partial y}\left[\frac{C_{000}^{\gamma}(x, y, z, t)}{P^{\gamma}(z, T)} \frac{\partial C_{00}(x, y, z, t)}{\partial y}\right] \\
& +D_{0 L} \frac{\partial}{\partial z}\left[\frac{C_{000}^{\gamma}(x, y, z, t)}{P^{\gamma}(z, T)} \frac{\partial C_{000}(x, y, z, t)}{\partial z}\right] ; \\
& \frac{\partial C_{020}(x, y, z, t)}{\partial t}=D_{0 L} \frac{\partial^{2} C_{020}(x, y, z, t)}{\partial x^{2}} . \\
& +D_{0 L} \frac{\partial^{2} C_{020}(x, y, z, t)}{\partial y^{2}}+D_{0 L} \frac{\partial^{2} C_{020}(x, y, z, t)}{\partial z^{2}} \\
& +D_{0 L} \frac{\partial}{\partial x}\left[C_{010}(x, y, z, t) \frac{C_{000}^{\gamma-1}(x, y, z, t)}{P^{\gamma}(z, T)} \frac{\partial C_{000}(x, y, z, t)}{\partial x}\right] \\
& +D_{0 L} \frac{\partial}{\partial y}\left[\frac{\partial C_{000}(x, y, z, t)}{\partial y} \times C_{010}(x, y, z, t) \frac{C_{00}^{\gamma-1}(x, y, z, t)}{P^{\gamma}(z, T)}\right] \\
& +D_{0 L} \frac{\partial}{\partial z}\left[C_{010}(x, y, z, t) \frac{C_{000}^{\gamma-1}(x, y, z, t)}{P^{\gamma}(z, T)} \frac{\partial C_{000}(x, y, z, t)}{\partial z}\right] \\
& +D_{0 C} \frac{\partial}{\partial x}\left[\frac{C_{00}^{\gamma}(x, y, z, t)}{P^{\gamma}(z, T)} \frac{\partial C_{010}(x, y, z, t)}{\partial x}\right] \\
& +D_{0 L} \frac{\partial}{\partial y}\left[\frac{C_{000}^{\gamma}(x, y, z, t)}{P^{\gamma}(z, T)} \frac{\partial C_{010}(x, y, z, t)}{\partial y}\right] \\
& +D_{0 L} \frac{\partial}{\partial z}\left[\frac{C_{000}^{\gamma}(x, y, z, t)}{P^{\gamma}(z, T)} \frac{\partial C_{010}(x, y, z, t)}{\partial z}\right]
\end{aligned}
$$




$$
\begin{aligned}
& \frac{\partial C_{001}(x, y, z, t)}{\partial t}=D_{0 L} \frac{\partial^{2} C_{001}(x, y, z, t)}{\partial x^{2}}+D_{0 L} \frac{\partial^{2} C_{001}(x, y, z, t)}{\partial y^{2}} \\
& +D_{0 L} \frac{\partial^{2} C_{001}(x, y, z, t)}{\partial z^{2}}+\Omega D_{0 S L} \frac{\partial}{\partial x}\left\{\left[1+\epsilon_{S L} g_{S L}(z, T)\right]\right. \\
& \left.\times \frac{\nabla_{S} \mu(x, y, z, t)}{k T}\left[1+\xi_{S} \frac{C_{000}^{\gamma}(x, y, z, t)}{P^{\gamma}(z, t)}\right] \int_{0}^{L_{z}} C_{000}(x, y, W, t) \mathrm{d} W\right\} \\
& +\Omega D_{0 S L} \frac{\partial}{\partial y}\left\{\left[1+\epsilon_{S L} g_{S L}(z, T)\right] \frac{\nabla_{S} \mu(x, y, z, t)}{k T}\right. \\
& \left.\times\left[1+\xi_{S} \frac{C_{000}^{\gamma}(x, y, z, t)}{P^{\gamma}(z, t)}\right] \int_{0}^{L_{z}} C_{000}(x, y, W, t) \mathrm{d} W\right\}
\end{aligned}
$$$$
\frac{\partial C_{002}(x, y, z, t)}{\partial t}=D_{0 L} \frac{\partial^{2} C_{002}(x, y, z, t)}{\partial x^{2}}
$$$$
+D_{0 L} \frac{\partial^{2} C_{002}(x, y, z, t)}{\partial y^{2}}+D_{0 L} \frac{\partial^{2} C_{002}(x, y, z, t)}{\partial z^{2}}
$$$$
+\Omega D_{0 S L} \frac{\partial}{\partial x}\left\{\left[1+\epsilon_{S L} g_{S L}(z, T)\right] \frac{\nabla_{S} \mu(x, y, z, t)}{k T}\right.
$$$$
\left.\times\left[1+\xi_{S} \frac{C_{000}^{\gamma}(x, y, z, t)}{P^{\gamma}(z, t)}\right] \int_{0}^{L_{z}} C_{001}(x, y, W, t) \mathrm{d} W\right\}
$$$$
+\Omega D_{0 S L} \frac{\partial}{\partial y}\left\{\left[1+\epsilon_{S L} g_{S L}(z, T)\right] \frac{\nabla_{S} \mu(x, y, z, t)}{k T}\right.
$$$$
\left.\times\left[1+\xi_{S} \frac{C_{000}^{\gamma}(x, y, z, t)}{P^{\gamma}(z, t)}\right] \int_{0}^{L_{z}} C_{001}(x, y, W, t) \mathrm{d} W\right\}
$$$$
+\Omega D_{0 S L} \frac{\partial}{\partial x}\left\{[ 1 + \epsilon _ { S L } g _ { S L } ( z , T ) ] \left[1+\xi_{S} C_{001}(x, y, z, t)\right.\right.
$$$$
\left.\times \frac{C_{000}^{\gamma-1}(x, y, z, t)}{P^{\gamma}(z, t)}\right] \int_{0}^{L_{z}} C_{000}(x, y, W, t) \mathrm{d} W .
$$$$
\left.\times \frac{\nabla_{S} \mu(x, y, z, t)}{k T}\right\}+\Omega D_{0 S L} \frac{\partial}{\partial y}\left\{\left[1+\epsilon_{S L} g_{S L}(z, T)\right]\right.
$$$$
\times\left[1+\xi_{S} C_{001}(x, y, z, t) \frac{C_{000}^{\gamma-1}(x, y, z, t)}{P^{\gamma}(z, t)}\right] .
$$$$
\left.\times \frac{\nabla_{S} \mu(x, y, z, t)}{k T} \int_{0}^{L_{z}} C_{000}(x, y, W, t) \mathrm{d} W\right\} ;
$$$$
\frac{\partial C_{110}(x, y, z, t)}{\partial t}=D_{0 L} \frac{\partial^{2} C_{110}(x, y, z, t)}{\partial x^{2}}+D_{0 L} \frac{\partial^{2} C_{110}(x, y, z, t)}{\partial y^{2}}
$$$$
+D_{0 L} \frac{\partial^{2} C_{110}(x, y, z, t)}{\partial z^{2}}+D_{0 L}\left\{\frac{\partial}{\partial x}\left[g_{L}(z, T) \frac{\partial C_{010}(x, y, z, t)}{\partial x}\right]\right.
$$$$
\left.+\frac{\partial}{\partial y}\left[g_{L}(z, T) \frac{\partial C_{010}(x, y, z, t)}{\partial y}\right]+\frac{\partial}{\partial z}\left[g_{L}(z, T) \frac{\partial C_{010}(x, y, z, t)}{\partial z}\right]\right\}
$$

$+D_{0 L}\left\{\frac{\partial}{\partial x}\left[\frac{C_{000}^{\gamma}(x, y, z, t)}{P^{\gamma}(z, t)} \frac{\partial C_{100}(x, y, z, t)}{\partial x}\right]\right.$

$+\frac{\partial}{\partial y}\left[\frac{C_{000}^{\gamma}(x, y, z, t)}{P^{\gamma}(z, t)} \frac{\partial C_{100}(x, y, z, t)}{\partial y}\right]$

$\left.+\frac{\partial}{\partial z}\left[\frac{C_{000}^{\gamma}(x, y, z, t)}{P^{\gamma}(z, t)} \frac{\partial C_{100}(x, y, z, t)}{\partial z}\right]\right\}$

$+D_{0 L}\left\{\frac{\partial}{\partial x}\left[C_{100}(x, y, z, t) \frac{C_{000}^{\gamma-1}(x, y, z, t)}{P^{\gamma}(z, t)} \frac{\partial C_{000}(x, y, z, t)}{\partial x}\right]\right.$

$+\frac{\partial}{\partial y}\left[C_{100}(x, y, z, t) \frac{C_{000}^{\gamma-1}(x, y, z, t)}{P^{\gamma}(z, t)} \frac{\partial C_{000}(x, y, z, t)}{\partial y}\right]$

$\left.+\frac{\partial}{\partial z}\left[C_{100}(x, y, z, t) \frac{\partial C_{000}(x, y, z, t)}{\partial z} \frac{C_{000}^{\gamma-1}(x, y, z, t)}{P^{\gamma}(z, t)}\right]\right\} ;$

$\frac{\partial C_{101}(x, y, z, t)}{\partial t}=D_{0 L} \frac{\partial^{2} C_{101}(x, y, z, t)}{\partial x^{2}}$

$+D_{0 L} \frac{\partial^{2} C_{101}(x, y, z, t)}{\partial y^{2}}+D_{0 L} \frac{\partial^{2} C_{101}(x, y, z, t)}{\partial z^{2}}+D_{0 L}$

$\times\left\{\frac{\partial}{\partial x}\left[g_{L}(z, T) \frac{\partial C_{000}(x, y, z, t)}{\partial x}\right]+\frac{\partial}{\partial y}\left[g_{L}(z, T)\right.\right.$

$\left.\left.\times \frac{\partial C_{000}(x, y, z, t)}{\partial y}\right]+\frac{\partial}{\partial z}\left[g_{L}(z, T) \frac{\partial C_{000}(x, y, z, t)}{\partial z}\right]\right\}$

$+\Omega D_{0 S L} \frac{\partial}{\partial x}\left\{\left[1+\epsilon_{S L} g_{S L}(z, T)\right] \frac{\nabla_{S} \mu(x, y, z, t)}{k T}\right.$

$\left.\times\left[1+\xi_{S} \frac{C_{000}^{\gamma}(x, y, z, t)}{P^{\gamma}(z, t)}\right] \int_{0}^{L_{z}} C_{100}(x, y, W, t) \mathrm{d} W\right\}$

$+\Omega D_{0 S L} \frac{\partial}{\partial y}\left\{\left[1+\epsilon_{S L} g_{S L}(z, T)\right] \frac{\nabla_{S} \mu(x, y, z, t)}{k T}\right.$

$\left.\times\left[1+\xi_{S} \frac{C_{000}^{\gamma}(x, y, z, t)}{P^{\gamma}(z, t)}\right] \int_{0}^{L_{z}} C_{100}(x, y, W, t) \mathrm{d} W\right\}$

$+\Omega D_{0 S L} \frac{\partial}{\partial x}\left\{\left[1+\epsilon_{S L} g_{S L}(z, T)\right]\left[1+\xi_{S} C_{100}(x, y, z, t)\right.\right.$

$\left.\left.\times \frac{C_{000}^{\gamma-1}(x, y, z, t)}{P^{\gamma}(z, t)}\right] \int_{0}^{L_{z}} C_{000}(x, y, W, t) \mathrm{d} W \frac{\nabla_{S} \mu(x, y, z, t)}{k T}\right\}$

$+\Omega D_{0 S L} \frac{\partial}{\partial y}\left\{\left[1+\epsilon_{S L} g_{S L}(z, T)\right]\left[1+\xi_{S} C_{100}(x, y, z, t)\right.\right.$

$\left.\left.\times \frac{C_{000}^{\gamma-1}(x, y, z, t)}{P^{\gamma}(z, t)}\right] \frac{\nabla_{S} \mu(x, y, z, t)}{k T} \int_{0}^{L_{z}} C_{000}(x, y, W, t) \mathrm{d} W\right\} ;$

$\frac{\partial C_{011}(x, y, z, t)}{\partial t}=D_{0 L} \frac{\partial^{2} C_{011}(x, y, z, t)}{\partial x^{2}}$

$+D_{0 L} \frac{\partial^{2} C_{011}(x, y, z, t)}{\partial y^{2}}+D_{0 L} \frac{\partial^{2} C_{011}(x, y, z, t)}{\partial z^{2}}$ 


$$
\begin{aligned}
& +D_{0 L} \frac{\partial}{\partial x}\left[\frac{C_{000}^{\gamma}(x, y, z, t)}{P^{\gamma}(z, T)} \frac{\partial C_{001}(x, y, z, t)}{\partial x}\right] \\
& +D_{0 L} \frac{\partial}{\partial y}\left[\frac{C_{000}^{\gamma}(x, y, z, t)}{P^{\gamma}(z, T)} \frac{\partial C_{001}(x, y, z, t)}{\partial y}\right] \\
& +D_{0 L} \frac{\partial}{\partial z}\left[\frac{C_{000}^{\gamma}(x, y, z, t)}{P^{\gamma}(z, T)} \frac{\partial C_{001}(x, y, z, t)}{\partial z}\right] \\
& +D_{0 L} \frac{\partial}{\partial x}\left[C_{001}(x, y, z, t) \frac{\partial C_{000}(x, y, z, t)}{\partial x} \times \frac{C_{000}^{\gamma-1}(x, y, z, t)}{P^{\gamma}(z, T)}\right] \\
& +D_{0 L} \frac{\partial}{\partial y}\left[C_{001}(x, y, z, t) \frac{C_{000}^{\gamma-1}(x, y, z, t)}{P^{\gamma}(z, T)} \frac{\partial C_{000}(x, y, z, t)}{\partial y}\right] \\
& +D_{0 L} \frac{\partial}{\partial z}\left[C_{001}(x, y, z, t) \frac{C_{000}^{\gamma-1}(x, y, z, t)}{P^{\gamma}(z, T)} \frac{\partial C_{000}(x, y, z, t)}{\partial z}\right] \\
& +\Omega D_{0 S L} \frac{\partial}{\partial x}\left\{\left[1+\xi_{S} \frac{C_{000}^{\gamma}(x, y, z, t)}{P \gamma(z, t)}\right]\right. \\
& \left.\times\left[1+\epsilon_{S L} g_{S L}(z, T)\right] \frac{\nabla_{S} \mu(x, y, z, t)}{k T} \int_{0}^{L_{z}} C_{010}(x, y, W, t) d W\right\} \\
& +\Omega D_{0 S L} \frac{\partial}{\partial y}\left\{\left[1+\xi_{S} \frac{C_{000}^{\gamma}(x, y, z, t)}{P^{\gamma}(z, t)}\right]\right. \\
& \left.\times\left[1+\epsilon_{S L} g_{S L}(z, T)\right] \frac{\nabla_{S} \mu(x, y, z, t)}{k T} \int_{0}^{L_{z}} C_{010}(x, y, W, t) d W\right\} \\
& +\Omega D_{0 S L} \frac{\partial}{\partial x}\left\{\int_{0}^{L_{z}} C_{000}(x, y, W, t) \mathrm{d} W\right. \\
& \times\left[1+\epsilon_{S L} g_{S L}(z, T)\right] \frac{\nabla_{S} \mu(x, y, z, t)}{k T} \\
& \left.\times\left[1+\xi_{S} C_{010}(x, y, z, t) \frac{C_{000}^{\gamma-1}(x, y, z, t)}{P^{\gamma}(z, t)}\right]\right\} \\
& +\frac{\partial}{\partial y}\left\{\frac{\nabla_{S} \mu(x, y, z, t)}{k T} \times\left[1+\epsilon_{S L} g_{S L}(z, T)\right]\right. \\
& \times\left[1+\xi_{S} C_{010}(x, y, z, t) \frac{C_{000}^{\gamma-1}(x, y, z, t)}{P^{\gamma}(z, t)}\right] \\
& \left.\times \int_{0}^{L_{z}} C_{000}(x, y, W, t) \mathrm{d} W\right\} \Omega D_{0 S L}
\end{aligned}
$$

Corrections of the first- and the second-orders to zero-order approximation of dopant concentration are

$$
\begin{gathered}
C_{i 00}(x, y, z, t)=-\frac{2 \pi D_{0 L}}{L_{x}^{2} L_{y} L_{z}} \sum_{n=0}^{\infty} n F_{n C} C_{n}(x, y, z) e_{n C}(t) \\
\times \int_{0}^{t} e_{n C}(-\tau) \int_{0}^{L_{x}} s_{n}(u) \int_{0}^{L_{y}} c_{n}(v) \int_{0}^{L_{z}} c_{n}(w) g_{L}(w, T)
\end{gathered}
$$

$$
\begin{aligned}
& \times \frac{\partial C_{i-100}(u, v, w, \tau)}{\partial u} \mathrm{~d} w \mathrm{~d} v \mathrm{~d} u \mathrm{~d} \tau \\
& -\frac{2 \pi D_{0 L}}{L_{x} L_{y}^{2} L_{z}} \sum_{n=0}^{\infty} n F_{n C} C_{n}(x, y, z) e_{n C}(t)
\end{aligned}
$$$$
\times \int_{0}^{t} e_{n C}(-\tau) \int_{0}^{L_{x}} c_{n}(u) \int_{0}^{L_{y}} s_{n}(v)
$$$$
\times \int_{0}^{L_{z}} c_{n}(w) g_{L}(w, T) \frac{\partial C_{i-100}(u, v, w, \tau)}{\partial v} \mathrm{~d} w \mathrm{~d} v \mathrm{~d} u \mathrm{~d} \tau
$$$$
-\frac{2 \pi D_{0 L}}{L_{x} L_{y} L_{z}^{2}} \sum_{n=0}^{\infty} n F_{n C} C_{n}(x, y, z) e_{n C}(t) \int_{0}^{t} e_{n C}(-\tau)
$$$$
\times \int_{0}^{L_{x}} c_{n}(u) \int_{0}^{L_{y}} c_{n}(v) \int_{0}^{L_{z}} s_{n}(w) g_{L}(w, T)
$$$$
\times \frac{\partial C_{i-100}(u, v, w, \tau)}{\partial w} \mathrm{~d} w \mathrm{~d} v \mathrm{~d} u \mathrm{~d} \tau, i \geq 1 ;
$$

$$
C_{010}(x, y, z, t)=-2 \pi \frac{D_{0 L}}{L_{x}^{2} L_{y} L_{z}} \sum_{n=0}^{\infty} n F_{n C} C_{n}(x, y, z) e_{n C}(t)
$$$$
\times \int_{0}^{t} e_{n C}(-\tau) \int_{0}^{L_{x}} s_{n}(u) \int_{0}^{L_{y}} \int_{0}^{L_{z}} \frac{C_{000}^{\gamma}(u, v, w, \tau)}{P^{\gamma}(w, T)}
$$$$
\times c_{n}(w) \frac{\partial C_{000}(u, v, w, \tau)}{\partial u} \mathrm{~d} w c_{n}(v) \mathrm{d} v \mathrm{~d} u \mathrm{~d} \tau
$$$$
-\frac{2 \pi D_{0 L}}{L_{x} L_{y}^{2} L_{z}} \sum_{n=0}^{\infty} n F_{n C} C_{n}(x, y, z) e_{n C}(t)
$$$$
\times \int_{0}^{t} e_{n C}(-\tau) \int_{0}^{L_{x}} c_{n}(u) \int_{0}^{L_{y}} s_{n}(v) \int_{0}^{L_{z}} c_{n}(w)
$$$$
\times \frac{C_{000}^{\gamma}(u, v, w, \tau)}{P^{\gamma}(w, T)} \frac{\partial C_{000}(u, v, w, \tau)}{\partial v} \mathrm{~d} w \mathrm{~d} v \mathrm{~d} u \mathrm{~d} \tau
$$$$
-2 \pi \frac{D_{0 C}}{L_{x} L_{y} L_{z}^{2}} \sum_{n=0}^{\infty} n F_{n C} c_{n}(x) c_{n}(y) c_{n}(z) e_{n C}(t)
$$$$
\times \int_{0}^{t} e_{n C}(-\tau) \int_{0}^{L_{x}} c_{n}(u) \int_{0}^{L_{y}} c_{n}(v) \int_{0}^{L_{z}} s_{n}(w)
$$$$
\times \frac{C_{000}^{\gamma}(u, v, w, \tau)}{P^{\gamma}(w, T)} \frac{\partial C_{000}(u, v, w, \tau)}{\partial w} \mathrm{~d} w \mathrm{~d} \nu \mathrm{d} u \mathrm{~d} \tau ;
$$

$$
\begin{gathered}
C_{020}(x, y, z, t)=-\frac{2 \pi D_{0 L}}{L_{x}^{2} L_{y} L_{z}} \sum_{n=0}^{\infty} n F_{n C} C_{n}(x, y, z) e_{n C}(t) \\
\times \int_{0}^{t} e_{n C}(-\tau) \int_{0}^{L_{x}} s_{n}(u) \int_{0}^{L_{y}} c_{n}(v) \int_{0}^{L_{z}} \frac{C_{000}^{\gamma-1}(u, v, w, \tau)}{P \gamma(w, T)}
\end{gathered}
$$




$$
\begin{aligned}
& \times c_{n}(w)\left[C_{010}(u, v, w, \tau) \frac{\partial C_{000}(u, v, w, \tau)}{\partial u}\right. \\
& \left.+C_{000}(u, v, w, \tau) \frac{\partial C_{010}(u, v, w, \tau)}{\partial u}\right] \mathrm{d} w \mathrm{~d} v \mathrm{~d} u \mathrm{~d} \tau-\frac{2 \pi D_{0 L}}{L_{x} L_{y}^{2} L_{z}} \\
& \times \sum_{n=0}^{\infty} n F_{n C} C_{n}(x, y, z)(z) e_{n C}(t) \int_{0}^{t} e_{n C}(-\tau) \int_{0}^{L_{x}} c_{n}(u) \\
& \times \int_{0}^{L_{y}} s_{n}(v) \int_{0}^{L_{z}} c_{n}(w) \frac{C_{000}^{\gamma-1}(u, v, w, \tau)}{P^{\gamma}(w, T)}\left[\frac{\partial C_{000}(u, v, w, \tau)}{\partial v}\right. \\
& \left.\times C_{010}(u, v, w, \tau)+C_{000}(u, v, w, \tau) \frac{\partial C_{010}(u, v, w, \tau)}{\partial v}\right] \\
& \times \mathrm{d} w \mathrm{~d} v \mathrm{~d} u \mathrm{~d} \tau-\frac{2 \pi D_{0 L}}{L_{x} L_{y} L_{z}^{2}} \sum_{n=0}^{\infty} n C_{n}(x, y, z) e_{n C}(t) \\
& \times F_{n C} \int_{0}^{t} e_{n C}(-\tau) \int_{0}^{L_{x}} c_{n}(u) \int_{0}^{L_{y}} c_{n}(v) \int_{0}^{L_{z}} \\
& \times\left[C_{010}(u, v, w, \tau) \frac{\partial C_{00}(u, v, w, \tau)}{\partial w}\right. \\
& \left.+C_{000}(u, v, w, \tau) \frac{\partial C_{010}(u, v, w, \tau)}{\partial w}\right] \\
& \times s_{n}(w) \frac{C_{000}^{\gamma-1}(u, v, w, \tau)}{P^{\gamma}(w, T)} \mathrm{d} w \mathrm{~d} v \mathrm{~d} u \mathrm{~d} \tau \\
& C_{001}(x, y, z, t)=-\frac{2 \pi D_{0 L} \Omega}{L_{x}^{2} L_{y} L_{z}} \sum_{n=0}^{\infty} n F_{n C} C_{n}(x, y, z) e_{n C}(t) \\
& \times \int_{0}^{t} e_{n C}(-\tau) \int_{0}^{L_{x}} s_{n}(u) \int_{0}^{L_{y}} \int_{0}^{L_{z}} \frac{\nabla_{S} \mu(u, v, w, \tau)}{k T} \\
& \times c_{n}(w)\left[1+\epsilon_{S L} g_{S L}(w, \tau)\right]\left[1+\xi_{S} \frac{C_{000}^{\gamma}(u, v, w, \tau)}{P^{\gamma}(w, \tau)}\right] \\
& \times \int_{0}^{L_{z}} C_{000}(u, v, W, \tau) \mathrm{d} W \mathrm{~d} w c_{n}(v) \mathrm{d} v \mathrm{~d} u \mathrm{~d} \tau-\frac{2 \pi D_{0 L} \Omega}{L_{x} L_{y}^{2} L_{z}} \\
& \times \sum_{n=0}^{\infty} n F_{n C} C_{n}(x, y, z) e_{n C}(t) \\
& \times \int_{0}^{t} e_{n C}(-\tau) \int_{0}^{L_{x}} c_{n}(u) \int_{0}^{L_{y}} c_{n}(v) \\
& \times \int_{0}^{L_{z}} \frac{\nabla_{S} \mu(u, v, w, \tau)}{k T}\left[1+\epsilon_{S L} g_{S L}(w, \tau)\right] \\
& \times c_{n}(w)\left[1+\xi_{S} \frac{C_{000}^{\gamma}(u, v, w, \tau)}{P^{\gamma}(w, \tau)}\right] \\
& \times \int_{0}^{L_{z}} C_{000}(u, v, W, \tau) \mathrm{d} W \mathrm{~d} w \mathrm{~d} v \mathrm{~d} u \mathrm{~d} \tau \\
& C_{002}(x, y, z, t)=-\frac{2 \pi D_{0 L} \Omega}{L_{x}^{2} L_{y} L_{z}} \sum_{n=0}^{\infty} n F_{n C} C_{n}(x, y, z) e_{n C}(t) \\
& \times \int_{0}^{t} e_{n C}(-\tau) \int_{0}^{L_{x}} s_{n}(u) \int_{0}^{L_{y}} \int_{0}^{L_{z}} \frac{\nabla_{S} \mu(u, v, w, \tau)}{k T} \\
& \times c_{n}(w)\left[1+\epsilon_{S L} g_{S L}(w, \tau)\right]\left[1+\xi_{S} \frac{C_{000}^{\gamma}(u, v, w, \tau)}{P^{\gamma}(w, \tau)}\right] \\
& \times \int_{0}^{L_{z}} C_{001}(u, v, W, \tau) \mathrm{d} W \mathrm{~d} w c_{n}(v) \mathrm{d} v \mathrm{~d} u \mathrm{~d} \tau-\frac{2 \pi D_{0 L} \Omega}{L_{x} L_{y}^{2} L_{z}} \\
& \times \sum_{n=0}^{\infty} n F_{n C} C_{n}(x, y, z) e_{n C}(t) \int_{0}^{t} e_{n C}(-\tau) \int_{0}^{L_{x}} c_{n}(u) \\
& \times \int_{0}^{L_{y}} c_{n}(v) \int_{0}^{L_{z}} \frac{\nabla_{S} \mu(u, v, w, \tau)}{k T}\left[1+\epsilon_{S L} g_{S L}(w, \tau)\right] \\
& \times c_{n}(w)\left[1+\xi_{S} \frac{C_{000}^{\gamma}(u, v, w, \tau)}{P \gamma(w, \tau)}\right] \int_{0}^{L_{z}} C_{001}(u, v, W, \tau) \\
& \times \mathrm{d} W \mathrm{~d} w \mathrm{~d} v \mathrm{~d} u \mathrm{~d} \tau-\frac{2 \pi D_{0 L} \Omega}{L_{x}^{2} L_{y} L_{z}} \sum_{n=0}^{\infty} n F_{n C} C_{n}(x, y, z) \\
& \times e_{n C}(t) \int_{0}^{t} e_{n C}(-\tau) \int_{0}^{L_{x}} s_{n}(u) \int_{0}^{L_{y}} c_{n}(v) \int_{0}^{L_{z}} \frac{\nabla_{S} \mu(u, v, w, \tau)}{k T} \\
& \times\left[1+\xi_{S} C_{001}(u, v, w, \tau) \frac{C_{000}^{\gamma-1}(u, v, w, \tau)}{P^{\gamma}(w, \tau)}\right] \\
& \times c_{n}(w)\left[1+\epsilon_{S L} g_{S L}(w, \tau)\right] \int_{0}^{L_{z}} C_{000}(u, v, W, \tau) d W \mathrm{~d} w \mathrm{~d} v \mathrm{~d} u \mathrm{~d} \tau \\
& \times-\frac{2 \pi D_{0 L} \Omega}{L_{x} L_{y}^{2} L_{z}} \sum_{n=0}^{\infty} n F_{n C} C_{n}(x, y, z) e_{n C}(t) \\
& \times \int_{0}^{t} e_{n C}(-\tau) \int_{0}^{L_{x}} c_{n}(u) \int_{0}^{L_{y}} c_{n}(v) \int_{0}^{L_{z}}\left[1+\epsilon_{S L} g_{S L}(w, \tau)\right] \\
& \times\left[1+\xi_{S} C_{001}(u, v, w, \tau) \frac{C_{000}^{\gamma-1}(u, v, w, \tau)}{P \gamma(w, \tau)}\right] \\
& \times \frac{\nabla_{S} \mu(u, v, w, \tau)}{k T} \int_{0}^{L_{z}} C_{000}(u, v, W, \tau) d W \mathrm{~d} w \mathrm{~d} v \mathrm{~d} u \mathrm{~d} \tau ; \\
& C_{110}(x, y, z, t)=-\frac{2 \pi D_{0 L}}{L_{x}^{2} L_{y} L_{z}} \sum_{n=0}^{\infty} n F_{n C} C_{n}(x, y, z) e_{n C}(t) \\
& \times \int_{0}^{t} e_{n C}(-\tau) \int_{0}^{L_{x}} s_{n}(u) \int_{0}^{L_{y}} c_{n}(v) \int_{0}^{L_{z}} c_{n}(w)
\end{aligned}
$$




$$
\begin{aligned}
& \times\left[g_{L}(w, T) \frac{\partial C_{010}(u, v, w, \tau)}{\partial u}+\frac{C_{000}^{\gamma}(u, v, w, \tau)}{P^{\gamma}(w, T)}\right. \\
& \times \frac{\partial C_{100}(u, v, w, \tau)}{\partial u}+\frac{C_{000}^{\gamma-1}(u, v, w, \tau)}{P^{\gamma}(w, T)} \frac{\partial C_{000}(u, v, w, \tau)}{\partial u} \\
& \times C_{100}(u, v, w, \tau)+g_{L}(w, T) \\
& \left.\times \frac{C_{000}^{\gamma}(u, v, w, \tau)}{P^{\gamma}(w, T)} \frac{\partial C_{00}(u, v, w, \tau)}{\partial u}\right] \mathrm{d} w \mathrm{~d} \nu \mathrm{d} u \mathrm{~d} \tau \\
& -\frac{2 \pi D_{0 L}}{L_{x} L_{y}^{2} L_{z}} \sum_{n=0}^{\infty} n F_{n C} C_{n}(x) \\
& \times e_{n C}(t) \int_{0}^{t} e_{n C}(-\tau) \int_{0}^{L_{x}} c_{n}(u) \int_{0}^{L_{y}} s_{n}(v) \int_{0}^{L_{z}} c_{n}(w) \\
& \times\left[g_{L}(w, T) \frac{\partial C_{010}(u, v, w, \tau)}{\partial v}+\frac{C_{000}^{\gamma}(u, v, w, \tau)}{P^{\gamma}(w, T)}\right. \\
& \times \frac{\partial C_{100}(u, v, w, \tau)}{\partial v}+\frac{C_{000}^{\gamma-1}(u, v, w, \tau)}{P^{\gamma}(w, T)} \frac{\partial C_{000}(u, v, w, \tau)}{\partial v} \\
& \times C_{100}(u, v, w, \tau)+g_{L}(w, T) \frac{C_{000}^{\gamma}(u, v, w, \tau)}{P^{\gamma}(w, T)} \\
& \left.\times \frac{\partial C_{000}(u, v, w, \tau)}{\partial v}\right] \mathrm{d} w \mathrm{~d} v \mathrm{~d} u \mathrm{~d} \tau \\
& -\frac{2 \pi D_{0 L}}{L_{x} L_{y} L_{z}^{2}} \sum_{n=0}^{\infty} n F_{n C} C_{n}(x, y, z) e_{n C}(t) \int_{0}^{t} e_{n C}(-\tau) \int_{0}^{L_{x}} c_{n}(u) \\
& \times \int_{0}^{L_{y}} c_{n}(v) \int_{0}^{L_{z}} s_{n}(w)\left[g_{L}(w, T) \frac{\partial C_{01}(u, v, w, \tau)}{\partial w}\right. \\
& +\frac{C_{000}^{\gamma}(u, v, w, \tau)}{P^{\gamma}(w, T)} \frac{\partial C_{100}(u, v, w, \tau)}{\partial w}+\frac{C_{000}^{\gamma-1}(u, v, w, \tau)}{P^{\gamma}(w, T)} \\
& \times \frac{\partial C_{000}(u, v, w, \tau)}{\partial w} C_{100}(u, v, w, \tau)+g_{L}(w, T) \\
& \left.\times \frac{C_{000}^{\gamma}(u, v, w, \tau)}{P^{\gamma}(w, T)} \frac{\partial C_{000}(u, v, w, \tau)}{\partial w}\right] \mathrm{d} w \mathrm{~d} v \mathrm{~d} u \mathrm{~d} \tau ; \\
& C_{101}(x, y, z, t)=-\frac{2 \pi D_{0 L}}{L_{x}^{2} L_{y} L_{z}} \sum_{n=0}^{\infty} n F_{n C} C_{n}(x, y, z) e_{n C}(t) \\
& \times \int_{0}^{t} e_{n C}(-\tau) \int_{0}^{L_{x}} s_{n}(u) \int_{0}^{L_{y}} c_{n}(v) \int_{0}^{L_{z}} c_{n}(w) g_{L}(w, T) \\
& \times \frac{\partial C_{000}(u, v, w, \tau)}{\partial u} \mathrm{~d} w \mathrm{~d} v \mathrm{~d} u \mathrm{~d} \tau-\frac{2 \pi D_{0 L}}{L_{x} L_{y}^{2} L_{z}} \sum_{n=0}^{\infty} n F_{n C} C_{n} \\
& \times(x, y, z) e_{n C}(t) \int_{0}^{t} e_{n C}(-\tau) \int_{0}^{L_{x}} c_{n}(u) \int_{0}^{L_{y}} s_{n}(v) \\
& \times \int_{0}^{L_{z}} c_{n}(w) g_{L}(w, T) \frac{\partial C_{000}(u, v, w, \tau)}{\partial v} \mathrm{~d} w \mathrm{~d} v \mathrm{~d} u \mathrm{~d} \tau \\
& -\frac{2 \pi D_{0 L}}{L_{x} L_{y} L_{z}^{2}} \sum_{n=0}^{\infty} n F_{n C} C_{n}(x, y, z) e_{n C}(t) \int_{0}^{t} e_{n C}(-\tau) \\
& \times \int_{0}^{L_{x}} c_{n}(u) \int_{0}^{L_{y}} c_{n}(v) \int_{0}^{L_{z}} s_{n}(w) g_{L}(w, T) \int_{0}^{L_{z}} c_{n}(w) g_{L}(w, T) \\
& \times \frac{\partial C_{000}(u, v, w, \tau)}{\partial w} \mathrm{~d} w \mathrm{~d} v \mathrm{~d} u \mathrm{~d} \tau-\frac{2 \pi D_{0 L} \Omega}{L_{x}^{2} L_{y} L_{z}} \sum_{n=0}^{\infty} n \\
& \times F_{n C} C_{n}(x, y, z) e_{n C}(t) \int_{0}^{t} e_{n C}(-\tau) \int_{0}^{L_{x}} s_{n}(u) \int_{0}^{L_{y}} c_{n}(v) \int_{0}^{L_{z}} c_{n}(w) \\
& \times\left[1+\epsilon_{S L} g_{S L}(w, \tau)\right]\left[1+\xi_{S} \frac{C_{000}^{\gamma}(u, v, w, \tau)}{P^{\gamma}(w, \tau)}\right] \\
& \times \frac{\nabla_{S} \mu(u, v, w, \tau)}{k T} \int_{0}^{L_{z}} C_{100}(u, v, W, \tau) \mathrm{d} W \mathrm{~d} w \mathrm{~d} v \mathrm{~d} u \mathrm{~d} \tau \\
& -\frac{2 \pi D_{0 L} \Omega}{L_{x} L_{y}^{2} L_{z}} \sum_{n=0}^{\infty} n C_{n}(x, y, z) e_{n C}(t) \int_{0}^{t} e_{n C}(-\tau) \\
& \times F_{n C} \int_{0}^{L_{x}} c_{n}(u) \int_{0}^{L_{y}} s_{n}(v) \int_{0}^{L_{z}}\left[1+\epsilon_{S L} g_{S L}(w, \tau)\right] \frac{\nabla_{S} \mu(u, v, w, \tau)}{k T} \\
& \times\left[1+\xi_{S} \frac{C_{000}^{\gamma}(u, v, w, \tau)}{P^{\gamma}(w, \tau)}\right] \int_{0}^{L_{z}} C_{100}(u, v, W, \tau) \\
& \times c_{n}(w) \mathrm{d} W \mathrm{~d} w \mathrm{~d} v \mathrm{~d} u \mathrm{~d} \tau-\frac{2 \pi D_{0 L} \Omega}{L_{x}^{2} L_{y} L_{z}} \sum_{n=0}^{\infty} n F_{n C} C_{n}(x, y, z) e_{n C}(t) \\
& \times \int_{0}^{t} e_{n C}(-\tau) \int_{0}^{L_{x}} s_{n}(u) \int_{0}^{L_{y}} c_{n}(v) \int_{0}^{L_{z}} c_{n}(w) \\
& \times\left[1+\epsilon_{S L} g_{S L}(w, \tau)\right]\left[1+\xi_{S} C_{100}(u, v, w, \tau) \frac{C_{000}^{\gamma-1}(u, v, w, \tau)}{P^{\gamma}(w, \tau)}\right] \\
& \times \frac{\nabla_{S} \mu(u, v, w, \tau)}{k T} \int_{0}^{L_{z}} C_{000}(u, v, W, \tau) \mathrm{d} W \mathrm{~d} w \mathrm{~d} \nu \mathrm{d} u \mathrm{~d} \tau \\
& -\frac{2 \pi D_{0 L} \Omega}{L_{x} L_{y}^{2} L_{z}} \sum_{n=0}^{\infty} n F_{n C} C_{n}(x, y, z) e_{n C}(t) \int_{0}^{t} e_{n C}(-\tau) \int_{0}^{L_{x}} s_{n}(u) \\
& \times \int_{0}^{L_{y}} c_{n}(v) \int_{0}^{L_{z}} c_{n}(w)\left[1+\epsilon_{S L} g_{S L}(w, \tau)\right] \\
& \times\left[1+\xi_{S} C_{100}(u, v, w, \tau) \frac{\nabla_{S} \mu(u, v, w, \tau)}{k T} \frac{C_{000}^{\gamma-1}(u, v, w, \tau)}{P^{\gamma}(w, \tau)}\right] \\
& \times \int_{0}^{L_{z}} C_{000}(u, v, W, \tau) \mathrm{d} W \mathrm{~d} w \mathrm{~d} v \mathrm{~d} u \mathrm{~d} \tau
\end{aligned}
$$




$$
\begin{aligned}
& C_{011}(x, y, z, t)=-\frac{2 \pi D_{0 L}}{L_{x}^{2} L_{y} L_{z}} \sum_{n=0}^{\infty} n F_{n C} C_{n}(x, y, z) e_{n C}(t) \\
& \times \int_{0}^{t} e_{n C}(-\tau) \int_{0}^{L_{x}} s_{n}(u) \int_{0}^{L_{y}} c_{n}(v) \int_{0}^{L_{z}}\left[\frac{C_{000}^{\gamma}(u, v, w, \tau)}{P \gamma(w, T)}\right. \\
& \times \frac{\partial C_{001}(u, v, w, \tau)}{\partial u}+C_{001}(u, v, w, \tau) \\
& \left.\times \frac{C_{000}^{\gamma-1}(u, v, w, \tau)}{P^{\gamma}(w, T)} \frac{\partial C_{000}(u, v, w, \tau)}{\partial u}\right] c_{n}(w) \mathrm{d} w \mathrm{~d} v \mathrm{~d} u \mathrm{~d} \tau \\
& -\frac{2 \pi D_{0 L}}{L_{x} L_{y}^{2} L_{z}} \sum_{n=0}^{\infty} n F_{n C} C_{n}(x, y, z) e_{n C}(t) \int_{0}^{t} e_{n C}(-\tau) \int_{0}^{L_{x}} c_{n}(u) \\
& \times \int_{0}^{L_{y}} s_{n}(v) \int_{0}^{L_{z}} c_{n}(w)\left[\frac{C_{000}^{\gamma}(u, v, w, \tau)}{P^{\gamma}(w, T)} \frac{\partial C_{001}(u, v, w, \tau)}{\partial v}\right. \\
& \left.+C_{001}(u, v, w, \tau) \frac{C_{000}^{\gamma-1}(u, v, w, \tau)}{P^{\gamma}(w, T)} \frac{\partial C_{000}(u, v, w, \tau)}{\partial v}\right] \mathrm{d} w \mathrm{~d} v \mathrm{~d} u \mathrm{~d} \tau \\
& -\frac{2 \pi D_{0 L}}{L_{x} L_{y} L_{z}^{2}} \sum_{n=0}^{\infty} n F_{n C} C_{n}(x, y, z) e_{n C}(t) \\
& \times \int_{0}^{t} e_{n C}(-\tau) \int_{0}^{L_{x}} c_{n}(u) \int_{0}^{L_{y}} c_{n}(v) \int_{0}^{L_{z}} s_{n}(w) \\
& \times\left[\frac{C_{000}^{\gamma}(u, v, w, \tau)}{P^{\gamma}(w, T)} \frac{\partial C_{001}(u, v, w, \tau)}{\partial w}\right. \\
& \left.+C_{001}(u, v, w, \tau) \frac{C_{000}^{\gamma-1}(u, v, w, \tau)}{P^{\gamma}(w, T)} \frac{\partial C_{000}(u, v, w, \tau)}{\partial w}\right] \mathrm{d} w \mathrm{~d} v \mathrm{~d} u \mathrm{~d} \tau \\
& -\frac{2 \pi D_{0 L} \Omega}{L_{x}^{2} L_{y} L_{z}} \sum_{n=0}^{\infty} n F_{n C} C_{n}(x, y, z) e_{n C}(t) \int_{0}^{t} e_{n C}(-\tau) \int_{0}^{L_{x}} s_{n}(u) \\
& \times \int_{0}^{L_{y}} c_{n}(v) \int_{0}^{L_{z}}\left[1+\epsilon_{S L} g_{S L}(w, \tau)\right] \\
& \times\left[1+\xi_{S} C_{010}(u, v, w, \tau) \frac{C_{000}^{\gamma-1}(u, v, w, \tau)}{P^{\gamma}(w, \tau)}\right] \frac{\nabla_{S} \mu(u, v, w, \tau)}{k T} \\
& \times \int_{0}^{L_{z}} C_{000}(u, v, W, \tau) \mathrm{d} W c_{n}(w) \mathrm{d} w \mathrm{~d} v \mathrm{~d} u \mathrm{~d} \tau \\
& -\frac{2 \pi D_{0 L} \Omega}{L_{x} L_{y}^{2} L_{z}} \sum_{n=0}^{\infty} n F_{n C} C_{n}(x, y, z) e_{n C}(t) \int_{0}^{t} e_{n C}(-\tau) \int_{0}^{L_{x}} c_{n}(u) \\
& \times \int_{0}^{L_{y}} s_{n}(v) \int_{0}^{L_{z}} \frac{\nabla_{S} \mu(u, v, w, \tau)}{k T} c_{n}(w)\left[1+\epsilon_{S L} g_{S L}(w, \tau)\right] \\
& \times\left[1+\xi_{S} C_{010}(u, v, w, \tau) \frac{C_{000}^{\gamma-1}(u, v, w, \tau)}{P^{\gamma}(w, \tau)}\right]
\end{aligned}
$$

$$
\begin{aligned}
& \times \int_{0}^{L_{z}} C_{000}(u, v, W, \tau) \mathrm{d} W \mathrm{~d} w \mathrm{~d} v \mathrm{~d} u \mathrm{~d} \tau \\
& -\frac{2 \pi D_{0 L} \Omega}{L_{x}^{2} L_{y} L_{z}} \sum_{n=0}^{\infty} n F_{n C} C_{n}(x, y, z) e_{n C}(t) \int_{0}^{t} e_{n C}(-\tau) \\
& \times \int_{0}^{L_{x}} s_{n}(u) \int_{0}^{L_{y}} c_{n}(v) \int_{0}^{L_{z}} c_{n}(w)\left[1+\epsilon_{S L} g_{S L}(w, \tau)\right] \\
& \times \frac{\nabla_{S} \mu(u, v, w, \tau)}{k T}\left[1+\xi_{S} \frac{C_{000}^{\gamma}(u, v, w, \tau)}{P^{\gamma}(w, \tau)}\right] \\
& \times \int_{0}^{L_{z}} C_{010}(u, v, W, \tau) \mathrm{d} W \mathrm{~d} w \mathrm{~d} v \mathrm{~d} u \mathrm{~d} \tau \\
& -\frac{2 \pi D_{0 L} \Omega}{L_{x} L_{y}^{2} L_{z}} \sum_{n=0}^{\infty} C_{n}(x, y, z) \\
& \times n F_{n C} e_{n C}(t) \int_{0}^{t} e_{n C}(-\tau) \int_{0}^{L_{x}} c_{n}(u) \int_{0}^{L_{y}} s_{n}(v) \\
& \times \int_{0}^{L_{z}} c_{n}(w)\left[1+\epsilon_{S L} g_{S L}(w, \tau)\right]\left[1+\xi_{S} \frac{C_{000}^{\gamma}(u, v, w, \tau)}{P^{\gamma}(w, \tau)}\right] \\
& \times \frac{\nabla_{S} \mu(u, v, w, \tau)}{k T} \int_{0}^{L_{z}} C_{010}(u, v, W, \tau) \mathrm{d} W \mathrm{~d} w \mathrm{~d} v \mathrm{~d} u \mathrm{~d} \tau .
\end{aligned}
$$

\section{References}

Grebene AB (1983) Bipolar and MOS analogous integrated circuit design. Wiley, New York

Gotra ZYu (1991) Technology of microelectronic devices. Radio and communication, Moscow (in Russian)

Kuzmichev VE (1989) Laws and formulas of physics. Naukova Dumka, Kiev (in Russian)

Lachin VI, Savelov NS (2001) Electronics. Phoenix, Rostov-na-Donu (in Russian)

Landau LD, Lefshits EM (2001) Theoretical physics, vol 7. Theory of elasticity. Physmatlit, Moscow (in Russian)

Pankratov EL (2005) Influence of spatial, temporal and concentrational dependence of diffusion coefficient on dopant dynamics: optimization of annealing time. Phys Rev B 72(7):075201

Pankratov EL (2008) Redistribution of dopant during microwave annealing of a multilayer structure for production $p$ - $n$-junction. J Appl Phys 103(6):064320

Pankratov EL (2009) Local doping and optimal annealing of a mesh multilayer structure for decreasing of spatial dimentions of integrated $p$ - $n$-junctions. Nano 4(5):303

Pankratov EL (2010) Influence of mechanical stress in a multilayer structure on spatial distribution of dopants in implantedjunction and diffusion-junction rectifiers. Mod Phys Lett B 24(9):867

Pankratov EL, Spagnolo B (2005) Optimization of impurity profile for $p$ - $n$-junction in heterostructures. Eur Phys J B 46(1):15 
Shalimova KV (1985) Physics of semiconductors. Energoatomizdat, Moscow (in Russian)

Sokolov YuD (1955) About determination mechanical stress in hoisting ropes of mines. Appl Mech 1:23
Zhang YW, Bower AF (1999) Numerical simulation of island formation in a coherent strained epitaxial thin film system. J Mech Phys Solids 47:2273 